1 ERK-mediated Curvature Feedback Regulates Branching Morphogenesis in Lung

\title{
2 Epithelial Tissue
}

3

4 *Tsuyoshi Hirashima ${ }^{1,2,3}$ and Michiyuki Matsuda ${ }^{2,4,5}$

5

6 1. The Hakubi Center, Kyoto University, Kyoto, Japan

7 2. Laboratory of Bioimaging and Cell Signaling, Graduate School of Biostudies, Kyoto

8 University, Kyoto, Japan

9 3. Japan Science and Technology Agency, PRESTO, Kawaguchi, Japan

10 4. Department of Pathology and Biology of Diseases, Graduate School of Medicine, Kyoto

11 University, Kyoto, Japan

12 5. Institute for Integrated Cell-Material Sciences, Kyoto University, Kyoto, Japan

\section{ORCID}

16 TH: 0000-0001-7323-9627

17 MM: 0000-0002-5876-9969

20 *Corresponding author: Tsuyoshi Hirashima, Ph.D

22 The Hakubi Center/Graduate School of Biostudies, Kyoto University

23 Yoshida-Konoe-cho, Sakyo-ku, Kyoto 606-8501, Japan

24 Phone: $+81-75-753-4422$

25 hirashima.tsuyoshi2m@kyoto-u.ac.jp 


\section{Abstract}

28 Intricate branching patterns emerge in internal organs because of the repetitive presence of 29 simple deformations in epithelial tissues. During murine lung development, epithelial cells in 30 distal tips of a single tube require fibroblast growth factor (FGF) signals generated by their 31 surrounding mesenchyme to form repetitive tip bifurcations. However, it remains unknown 32 how the cells employ FGF signaling to convert their behaviors to achieve the recursive 33 branching processes. Here we show a self-sustained epithelial regulatory system during the 34 murine lung branching morphogenesis, mediated by extracellular signal-regulated kinase 35 (ERK), which acts as a downstream driver of FGF signaling. We found that tissue-scale 36 curvature regulates ERK activity in the lung epithelium using two-photon live cell imaging and 37 mechanical perturbations. ERK is activated specifically in epithelial tissues with a positive 38 curvature, regardless of whether the change in curvature was attributable to morphogenesis or 39 artificial perturbations. Moreover, we found that ERK activation accelerates actin 40 polymerization specifically at the apical side of cells, and mechanically contributes to the 41 extension of the apical membrane, leading to a decrease in epithelial tissue curvature. These 42 results indicate the existence of a negative feedback loop between tissue curvature and ERK 43 activity beyond scale. We confirmed that this regulation was sufficient to generate the recursive 44 branching processes by a mathematical model. Taken together, we propose that ERK mediates 45 the curvature feedback loop underlying the process of branching morphogenesis in developing 46 lungs.

48 Keywords: Branching morphogenesis, ERK, FRET cell imaging, lung development, 49 mathematical model, self-organization 


\section{Introduction}

52 Characteristic patterns are generated in developing tubular organs through the repeated

53 occurrence of tissue morphogenetic modes, such as elongation, bending, and bifurcation ${ }^{1-4}$.

54 During murine lung development, the complex branched architecture is stereotypically created

55 from a simple epithelial tube, by assembling morphogenetic modes as building motifs with a spatio-temporal order ${ }^{2}$. Previous genetic studies have revealed the essential molecules and signaling pathways underlying lung epithelial morphogenesis ${ }^{5-7}$. Moreover, previous studies have proposed how cellular behaviors and mechanical forces determine the tube shape ${ }^{8-10}$. Thus, the interplay between chemical signals and cell mechanics in constituent cells determines the organized periodic branched pattern, though it is not completely understood yet.

Several pharmacological and genetic studies have demonstrated that fibroblast growth factor (FGF) signaling is a key regulator of lung branching morphogenesis during murine development ${ }^{11}$. The treatment of an isolated epithelium in which the mesenchyme is removed with different FGF ligands has clarified the distinct roles of FGFs in lung epithelial morphology. For example, FGF1 and FGF10 induce bud formation, while FGF7 gives rise to a balloon-like shape, and any of these ligands could promote epithelial proliferation ${ }^{12-14}$. An investigation of the spatio-temporal expression pattern uncovered the fact that FgflO is expressed locally in the mesenchyme surrounding the distal bud of the lung epithelium ${ }^{14}$, and its main receptor, $F g f r 2 b$, is homogeneously expressed in the lung epithelium ${ }^{12}$. These results led to hypothesize that the Fgf10 prepattern initiates branching during lung development ${ }^{14}$; this was partially supported by results of previous studies on mice in which $F g f 10$ or its main receptor $F g f r 2 b$ were knocked out and showed the complete absence of lungs ${ }^{15-17}$. However, it was demonstrated that the ubiquitous overexpression of Fgfl0 in its knocked out background could restore the branching process ${ }^{18}$, indicating that the $\mathrm{FgflO}$ expression is required but the precise prepattern is dispensable for the murine lung branching morphogenesis. Thus, we explored how FGF signals are converted to cellular behaviors underlying tissue morphogenesis.

A main FGF downstream signaling pathway, the extracellular signal-regulated kinase ERK/MAP kinase pathway, includes a three-tiered kinase cascade, i.e., RAF - MEK - ERK ${ }^{11}$. The genetic inactivation of either Mek1/2 or Erk1/2 specifically in the airway epithelium results in lung agenesis ${ }^{19}$, implying that ERK and its activation were essential for lung development ${ }^{20}$. Previously, immunofluorescence staining results suggested that the phosphorylation of ERK was most likely to occur in the distal tips of the lung epithelium, and may thus be involved in

83 the budding process during lung development ${ }^{21-23}$. FGFR2-mediated ERK activation 
85 bundles, and promote cell migration within epithelial tissues ${ }^{22}$. These reports revealed the role

86 of ERK activity in cellular behavior during lung development. However, it is still unclear how

87 the epithelial cells collectively behave to generate the periodic branching morphogenesis

88 mediated via ERK signaling.

89 In this study, we show a physical basis for self-sustained branching morphogenesis by

90 combining two-photon live cell imaging of a Förster resonance energy transfer (FRET)-based

91 biosensor, mechanical perturbation, and mathematical modeling. We found that ERK

92 activation occurs specifically in epithelial tissues with a positive curvature, and promotes cell

93 extension through actin polymerization at the apical edges, resulting in a decreased tissue

94 curvature. Thus, our study clarifies that the negative feedback loop between the tissue curvature

95 and ERK activity underlies the process of repetitive branching morphogenesis in developing

96 murine lung epithelial tissues. 


\section{$98 \quad$ Results}

\section{1. ERK activation occurs in distal tips of lung epithelial tissue}

100 We first examined the impact of ERK activity on lung epithelial morphology during murine

101 development. We cultured developing murine lungs dissected at embryonic day (E) 12.5 in the

102 presence or absence of PD0325901, an inhibitor of the ERK activator MEK, under explant

103 culture conditions. The growth of 1-day cultured lung epithelial tissues was remarkably

104 impaired by the inhibition of ERK activity (Figure 1A, 1B), indicating the importance of ERK

105 activation for epithelial morphogenesis during this stage.

106 Then, we visualized the spatial distribution of ERK activity in lung lobes dissected from 107 murine embryos that ubiquitously expressed the FRET biosensor for the ERK activity ${ }^{24-26}$ via

108 whole tissue two-photon live imaging (Figure 1C, Movie 1). FRET images showed that the

109 ERK activity in the epithelium was highly dependent on tissue curvature; the ERK activity was

110 higher in the curved sheet protruding the mesenchyme (convex), while it was lower in the sheet

111 bent towards the lumen (concave) (Figure 1C). Although endothelial cells exhibited relatively

112 high levels of ERK activity, they were less involved in epithelial branching morphogenesis ${ }^{27}$;

113 thus, we focused on ERK activity in epithelial tissues. The basal surface of epithelial tissue

114 was extracted for quantification, and positions at nodes of generated meshes were embedded

115 on the surface, enabling us to map chemical and geometrical quantities, such as ERK activity,

116 epithelial tissue curvature, and tissue thickness (Figure 1D, S1A). As expected, there was a

117 positive correlation between the ERK activity and tissue curvature ( $\rho=0.51$, where $\rho$ is the

118 Spearman's rank correlation coefficient; Figure 1E, 1G, S1B), which indicates that ERK

119 activation would occur mainly in convex regions. Moreover, there was a negative correlation

120 between the ERK activity and tissue thickness ( $\rho=-0.48$; Figure $1 F, 1 G)$. As observed in distal

121 buds, the epithelial tissue was thinner in the tip region, where ERK was activated, than in the

122 stalk region, where ERK was inactivated (Figure 1H). These results suggest the existence of 123 interplay between the ERK activity and cell morphology in developing murine lungs. 
A

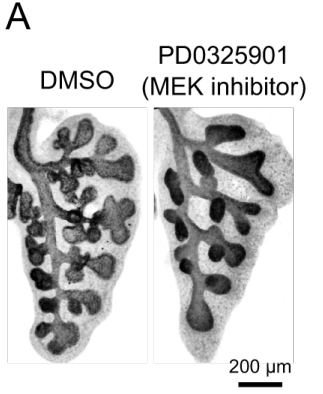

$\mathrm{E}$

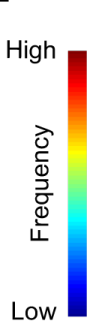

125
B

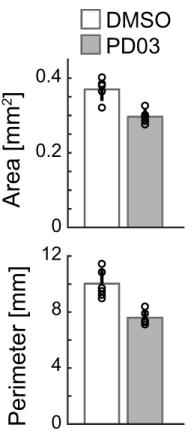

$\mathrm{F}$
C
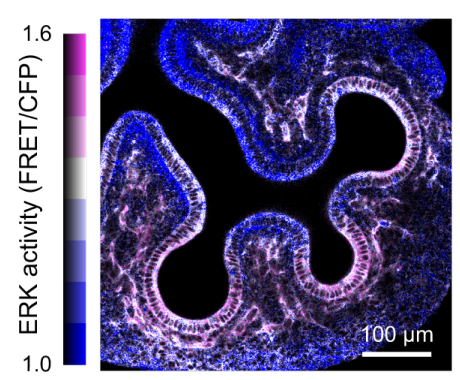

G
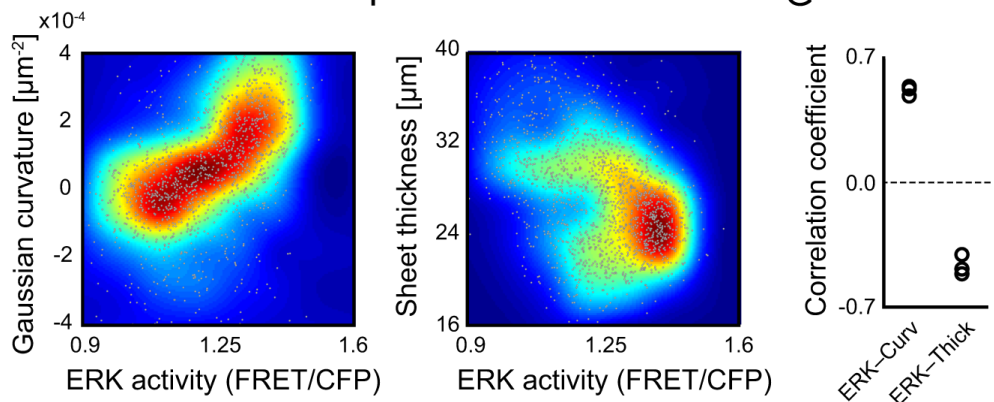

Figure 1 Relationship between ERK activity and tissue geometry in the lung epithelium

128 (A) Immunofluorescence images of anti-E-cadherin staining in an ex vivo culture of lungs for 1291 day from E12.5, after treatment with dimethyl sulfoxide (DMSO) (left) and $1 \mu$ M PD0325901 130 (right). Scale bar, $200 \mu \mathrm{m}$. (B) Area and perimeter of epithelial tissues from the projected 131 images. Data represent mean and standard deviation (SD) values. $\mathrm{N}=6$, two-sample t-test, $132 \mathrm{p}<0.001$.

133 (C) Representative ERK activity map in a developing lung at E12.5. Scale bar, $100 \mu \mathrm{m}$.

134 (D) Gaussian curvature map on the basal side of lung epithelial cells.

135 (E, F) Scatter plot of ERK activity versus Gaussian curvature (E) and that of ERK activity 136 versus sheet thickness (F). Color indicates frequency. $n=427, N=3$.

137 (G) Correlation coefficient of $(E)$ and $(F) . N=3$, one-sample t-test, $p=0.001(E)$, and $p=0.004$ 138 (F).

139 (H) ERK activity map in the distal region of a lung dissected at E12.5. Arrows facing each 140 other indicate the sheet thickness at a tip (magenta) and distal stalk (blue). Scale bar, $50 \mu \mathrm{m}$. 


\section{2. ERK activity follows the morphological change of isolated epithelial tissues}

143 To analyze how ERK activity is regulated in the lung epithelium, we isolated epithelial tissues

144 from mesenchymal tissues, and embedded them in Matrigel with culture media containing 145 FGFs for ex vivo culture (Figure 2A). This simplified experimental system allows us to explore 146 responses to external chemical and mechanical stimuli in epithelial cells under regulated 147 conditions. As reported previously ${ }^{12,13}$, treatment with $500 \mathrm{ng} \mathrm{mL}{ }^{-1}$ FGF1 for 1 day induces

148 branched epithelial buds, while that with $100 \mathrm{ng} \mathrm{mL}^{-1}$ FGF7 gives rise to a cyst-like structure 149 with an enlarged lumen (Figure 2B). PD0325901 treatment with FGF1 results in the collapse 150 of the lumen and absence of large deformations; this was also observed with the absence of 151 growth factors with 1-day-old culture (Figure 2B). We then examined the spatial distribution 152 of ERK activity in epithelial tissues, 1 hour after chemical treatment. FGF1 activates ERK 153 exclusively at the distal buds, in a manner similar to that observed with the mesenchyme 154 (Figure 1C), through ligand endocytosis from the basal side of cells (Figure S2, Movie 2), 155 despite the homogeneous expression of four FGF receptors, i.e., FGFR1, FGFR2, FGFR3, and 156 FGFR4 in the distal epithelial tissues ${ }^{12,18,28,29}$. In contrast, FGF7 activates the ERK almost homogeneously in the epithelial tissues (Figure 2C). As expected, ERK activity was homogeneously low in epithelial cells treated with PD0325901 or cultured without any growth factors (Figure 2C). These results suggest that localized ERK activation at the distal tips is key to the process of branching morphogenesis.

To examine the spatio-temporal dynamics of ERK activity during the branching of isolated epithelial tissues, we performed two-photon live imaging under FGF1 supplemented conditions. We found that the ERK activity increased during the protrusion of epithelial tips, and decreased when the tip apex became flat during the tip bifurcation process (Figure 2D, Movie 3). Notably, no mesenchymal cells were observed around the distal tip of the epithelium (Figure 2D), suggesting that tip bifurcation could occur in a self-sustaining manner without mesenchymal cells. The quantification of the ERK activity and tissue curvature at the tip apex shows that the decrease in ERK activity follows that in curvature, with an average delay of 97 min (Figure 2E, 2F). This observation suggests that the ERK activity is controlled by the curvature of the epithelial cells.

To clarify the morphological differences in cells between the convex and concave regions

172 of epithelial tissues, we measured the basal and apical areas of epithelial cells through whole-

173 tissue staining using E-cadherin. The basal cell area of isolated epithelial tissues in the convex 174 region is significantly larger than that in the concave region (Figure 2G, left), while the apical 175 cell area in the convex region is almost equivalent to that in the concave region (Figure $2 \mathrm{G}$, 
176 right). The epithelial basal edges act as an interface for cells to receive growth factor signals

177 from external environments that activate ERK (Figure S2). Therefore, we speculate that the

178 extension of basal membranes would trigger ERK activation in the convex regions of epithelial

179 tissues.

180

A

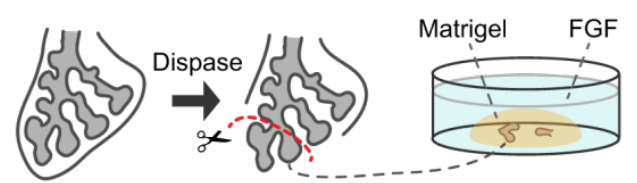

B

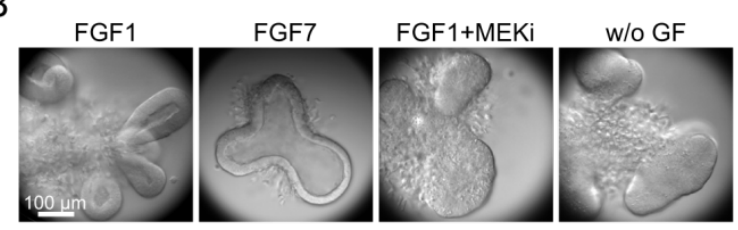

C

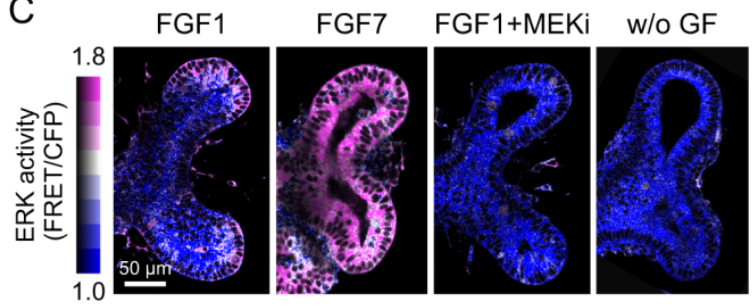

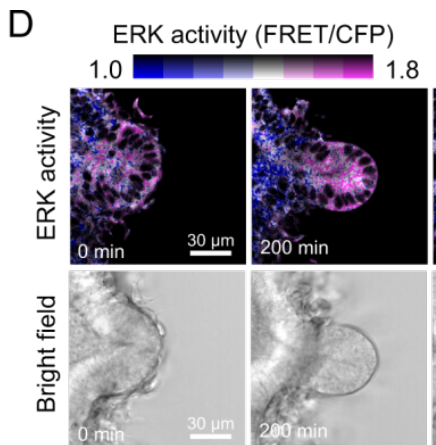

E

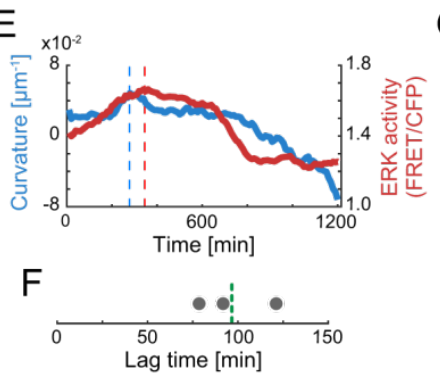

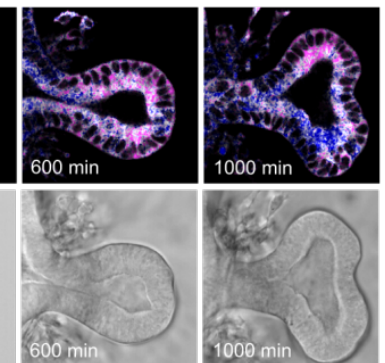

G Basal Apical

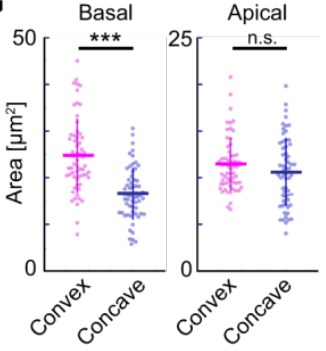

181

182

183 Figure 2 ERK activity and tissue curvature in isolated epithelial cells

184 (A) Schematics of the culture system for isolated lung epithelial tissues.

185 (B) Bright field images of isolated epithelial tissues subjected chemical treatments for 1 day 186 from E12.5. Concentrations: $500 \mathrm{ng} \mathrm{mL}^{-1} \mathrm{FGF} 1,100 \mathrm{ng} \mathrm{mL}{ }^{-1} \mathrm{FGF} 7,1 \mu \mathrm{M}$ PD0325901 (MEKi). 187 Scale bar, $100 \mu \mathrm{m}$.

188 (C) ERK activity of isolated epithelial tissues subjected to chemical treatments shown in (B)

189 for 1 hour from E12.5. Scale bar, $50 \mu \mathrm{m}$.

190 (D) Time-lapse images of ERK activity (upper) and bright field (lower) during terminal 191 bifurcation of lung epithelial tissues. Scale bar, $30 \mu \mathrm{m}$.

192 (E) Tissue curvature (blue) and ERK activity (red) at the tip apex over time (E). Vertical dotted 193 lines indicate the peak time for the tissue curvature and ERK activity, respectively.

194 (F) Lag time from the peak for tissue curvature to the peak for ERK activity. N=3.

195 (G) Basal area (left) and apical area (right) of individual cells in the convex and concave 196 regions of isolated epithelial tissues. Bold and error bars represent the mean and SD values, 197 respectively. $n=60, N=3$. Welch's t test, $\mathrm{p}<0.001$ (left) and $\mathrm{p}=0.18$ (right). 


\section{3. The shape of epithelial tissues controls the ERK activity}

200 To test whether the shape of epithelial tissues controls the ERK activity, we performed a

201 physical perturbation assay using isolated epithelial tissues. We embedded isolated epithelial 202 tissues within the FGF1-containing Matrigel filled inside a polydimethylsiloxane (PDMS) 203 chamber, and uniaxially compressed it by 33\% either parallelly or vertically to the distal204 proximal axis of the lung epithelium (Figure 3A). Time-lapse images obtained during this 205 process allow us to examine the ERK activity response to perturbations in different axes 206 (Figure 3B, 3C). For parallel compression, the curved epithelial tissues at the tip apex became 207 flattened (Figure 3D) and ERK activity decreased exclusively in the flat region (Figure 3E, 208 Movie 4). However, for vertical compression, the tip apex of the epithelium became sharp 209 (Figure 3D) and ERK activation followed the change in tissue curvature (Figure 3E, Movie 5). 210 We further examined the ERK activity response to cell deformation. The manipulation of 211 cell shape in curved epithelial tissues remains unfeasible under 3D culture conditions; thus, we 212 performed a strain perturbation assay under planar culture conditions using dissociated lung 213 epithelial cells (Figure 3F). Under planar culture conditions, the stretching of cells led to ERK 214 activation, while their compression led to ERK inactivation (Figure $3 \mathrm{G}, 3 \mathrm{H}$ ), indicating that 215 the strain on the cell area is key to curvature-dependent ERK activity regulation. Notably, the 216 surface area of the basal side of a cell is significantly larger in the convex region with high 217 ERK activity than in the concave region with lower ERK activity (Figure 2G). Together, 218 epithelial tissue curvature controls the ERK activity in cells, most likely through the strained 219 state of their basal membranes. 
A
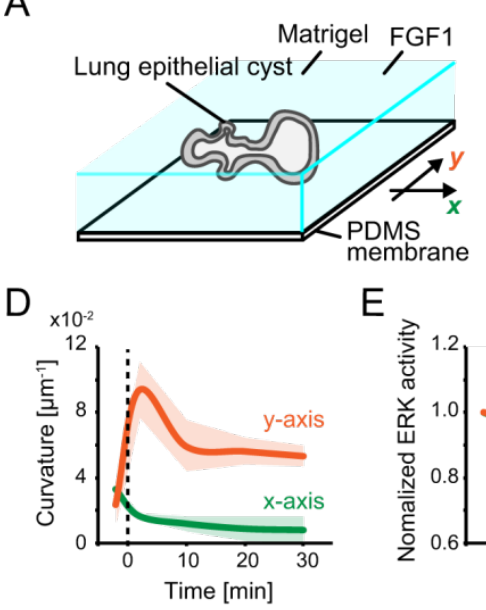

$\mathrm{F}$

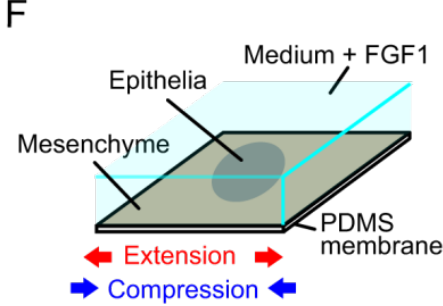

$\mathrm{E}$

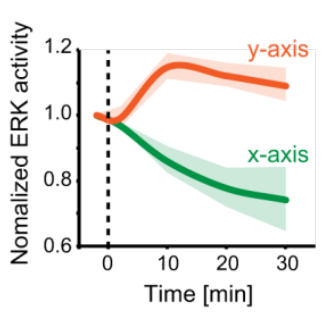

G

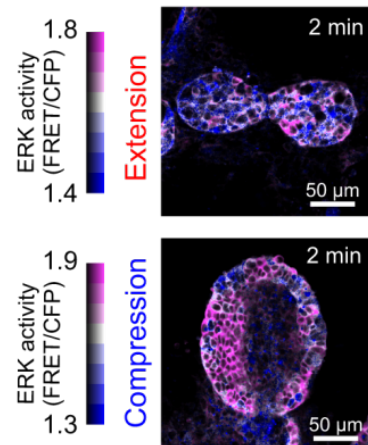

C
B
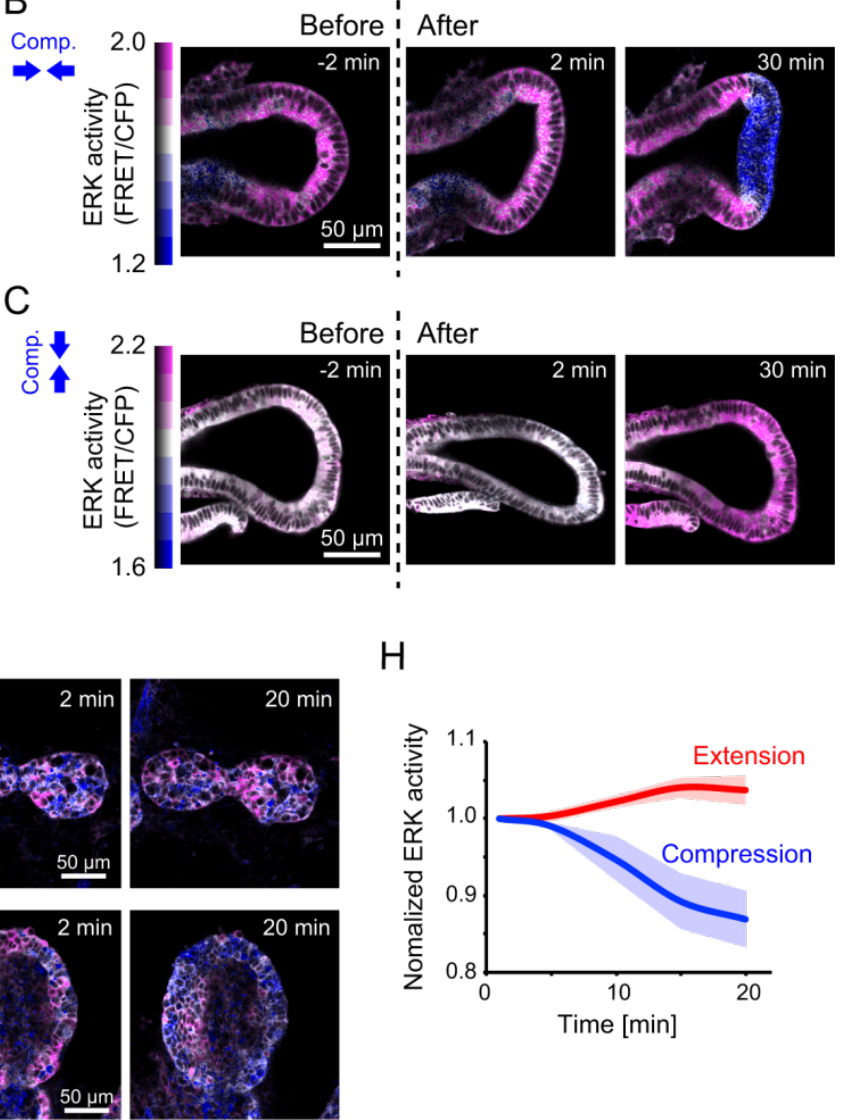

$\mathrm{H}$

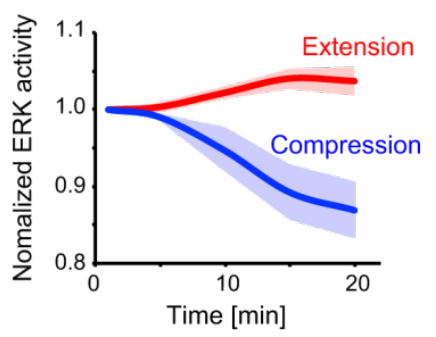

\section{Figure 3 Response of ERK activity to mechanical perturbation}

224 (A) Schematics of uniaxial compression assay performed on an isolated epithelium under 3D

225 culture conditions. The $x$ and $y$ axes indicate directions parallel and vertical to the distal and 226 proximal axes of the epithelium, respectively.

227 (B, C) Time-lapse images of the ERK activity for parallel compression (B) and vertical 228 compression (C). Time origin indicates the timing of mechanical compression. Scale bars, 50 $229 \mu \mathrm{m}$.

230 (D, E) Tissue curvature at the tip apex (D) and normalized ERK activity (E) over time. Colors 231 represent the axes of compression, as shown in (A). Data represent mean and SD values. N=3.

232 (F) Schematics of uniaxial mechanical perturbations under 2D culture conditions.

233 (G) Time-lapse images of the ERK activity in the isolated lung epithelium for the extension 234 (upper) and compression (lower) processes. Scale bar, $50 \mu \mathrm{m}$.

235 (H) Normalized ERK activity for the extension (red) and the compression (blue) processes over 236 time. Time origin indicates the timing of mechanical perturbations. Data represent mean and 237 SD values. $\mathrm{N}=3$. 
 \\ 4. ERK activation modulates cell shape via actin polymerization at the apical side}

240 Next, we investigate how the ERK activity contributes to epithelial morphology and mechanics.

241 First, we observed the change in the cell shape of isolated epithelial tissues treated with 242 PD0325901. Drug treatment led to an inhibition in the ERK activity within 30 min after 243 administration (Figure S3A), and the apico-basal length of epithelial cells became longer than 244 that observed in the control within 2 hours (Figure 4A, Movie 6). Hereafter, we refer to the 245 apico-basal length of cells as the cell height, and the cell length orthogonal to the apico-basal 246 axis as the cell width (Figure 4B). ERK inactivation in cells treated with PD0325901 lengthens the cell height to $124 \%$ and shortens the cell width to $92 \%$ on an average, despite no significant changes in the cell size, compared to mock-treated cells (Figure 4C). This trend is consistent with that observed in the dissected murine lungs (Figure 1E-G). These results indicate that the ERK activity regulates the cell height, while maintaining the cell size.

Next, to explore what causes the cell shape to change mechanically, we performed inhibitor assays on cytoskeletons, such as actin, microtubule, and non-muscle myosin, which allowed us to determine the mechanical contributions of targeted cytoskeletons to the cell morphology. Given that the cell size is maintained even if the cell shape varies (Figure 4C), two representative geometrical parameters, cell height and cell width, are in an inverse relationship (see Materials and Methods IIa). Thus, we focused only on the change in the cell height caused by cytoskeletal perturbations. The cell height was increased markedly upon treatment with Latrunculin A or Cytochalasin D, each an inhibitor of actin polymerization, as observed in inhibiting ERK activity with PD0325901; conversely, the cell height was decreased by treatment with Jasplakinolide, which promotes actin polymerization (Figure 4D, 4E). Because F-actin remarkably accumulates at the apical side of cells (Figure S3B), we speculated that actin polymerization at the apical edge would contribute to cell width extension, leading to the shortening of the cell height. The cell height was also shortened by interfering with microtubule polymerization with Nocodazole; combined treatment with Nocodazole and Latrunculin A resulted in significant cell height extension via the rupture of lateral cell edges (Figure S3D). Microtubules within cells are distributed along the apico-basal axis (Figure S3C), and would thus stabilize cytoskeletal architectures sustaining cell height with F-actin. The inhibition of the activity of non-muscle myosin II with Blebbistatin resulted in no significant difference in cell height, compared to that observed with DMSO control (Figure 4E). This was consistent with the fact that the activity of myosin is insignificant in the tips of lung epithelial tissue (Figure S3E-E"). To further analyze the mode of actin polymerization, we used CK-666, an Arp2/3 complex inhibitor CK-666, and SMIFH2, an inhibitor of the formin homology 2 
273 domain. We found that the cell height was lengthened for CK-666 treatment, but slightly

274 shortened for SMIFH2 treatment (Figure 4E), suggesting that actin polymerization would

275 depend on the Arp2/3-mediated nucleation of branched filaments, rather than formin-mediated

276 actin nucleation.

277 Because the inhibition of ERK activity and actin polymerization resulted in similar effects

278 on the cell height, we speculated that ERK activation promoted F-actin accumulation within

279 cells. Simultaneous imaging for the two factors revealed that F-actin accumulation at the apical

280 side of cells was observed exclusively in the tips where ERK activation occurs, under FGF1-

281 treated conditions (Figure 4F). Mapping between the ERK activity and F-actin accumulation

282 level via 3D whole-tissue imaging clearly showed a positive correlation with FGF1 ( $\rho=0.51)$,

283 although the inhibition of the ERK activity led to a significant decrease in F-actin accumulation

284 at the apical side of cells throughout the tissue, and relatively uniform distribution (Figure 4G).

285 In addition, treatment with FGF7, which activates ERK globally in the isolated epithelial

286 tissues, regardless of the tissue curvature (Figure 2C), led to an increase in the apical F-actin 287 accumulation overall throughout the epithelial tissues (Figure 4G). Thus, we found that ERK 288 activation promotes actin polymerization at the apical edges of cells, and ultimately controls 289 the cellular shape in the epithelial monolayer.

290

A

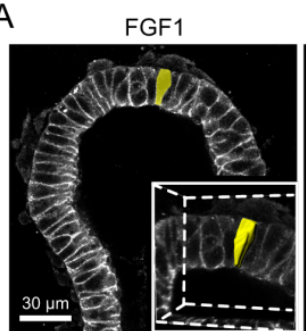

D

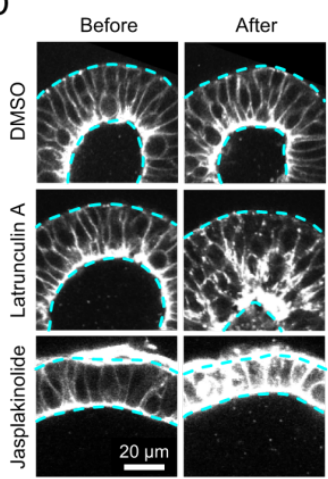

FGF1 + MEK inhibitor

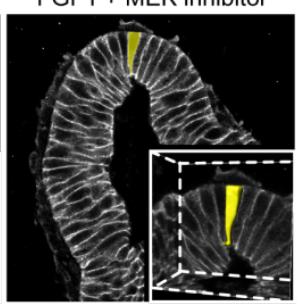

$\mathrm{E}$

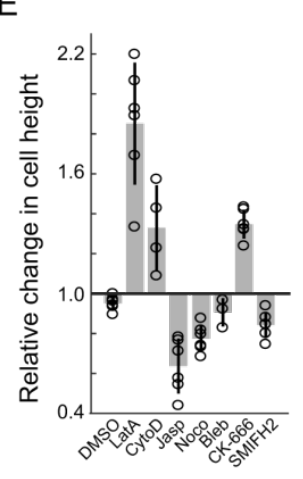

B

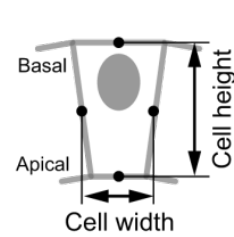

C
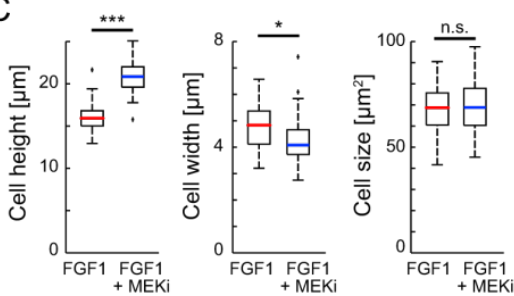

$\mathrm{F}$

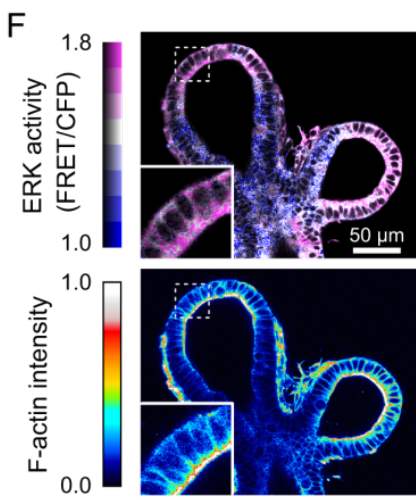

294 (A) Change in the cell shape of an isolated epithelium upon treatment with either $500 \mathrm{ng} \mathrm{mL}^{-1}$

295 FGF1 (left) or $500 \mathrm{ng} \mathrm{mL}^{-1} \mathrm{FGF} 1$ and $1 \mu \mathrm{M}$ PD0325901. Cell membranes were visualized 
296 using the whole-mount immunofluorescence of anti-E-cadherin. The inset windows represent 297 a magnified 3D rendering focusing on a representative single cell marked in yellow. Scale bar, $29830 \mu \mathrm{m}$.

299 (B) Schematics for cellular morphological measurement.

300 (C) Quantification of cell morphological parameters, including cell height, width, and size. $301 \mathrm{n}=60, \mathrm{~N}=3$. Welch's t test, $\mathrm{p}<0.001$ (left), $\mathrm{p}=0.013$ (middle), and $\mathrm{p}=0.4$ (right).

302 (D) Change in the cell morphology of an isolated epithelium upon treatment with either DMSO 303 as a control, $1 \mu \mathrm{M}$ Latrunculin A, or $1 \mu \mathrm{M}$ Jasplakinolide. The cell shape was visualized using 304 SiR-Actin, a F-actin labeling probe. Scale bar, $20 \mu \mathrm{m}$.

305 (E) Relative change in cell height upon inhibitor treatment for cytoskeletons. Concentrations 306 of inhibitors: $1 \mu \mathrm{M}$ Latrunculin $\mathrm{A}, 1 \mu \mathrm{M}$ Cytochalasin $\mathrm{D}, 1 \mu \mathrm{M}$ Nocodazole, $30 \mu \mathrm{M}$ 307 Blebbistatin, $1 \mu \mathrm{M}$ Jasplakinolide, $100 \mu \mathrm{M}$ CK-666, and $10 \mu \mathrm{M}$ SMIFH2. The bar graph and 308 error bars represent mean and SD values. $\mathrm{N} \geq 3$ for each treatment.

309 (F) Spatial map of ERK activity and F-actin accumulation level visualized by SiR-Actin 310 labeling upon treatment with $500 \mathrm{ng} \mathrm{mL}^{-1} \mathrm{FGF}$. Inset windows show magnified views that 311 focus on their subcellular localization. Scale bar, $50 \mu \mathrm{m}$.

312 (G) Scatter plot and contour map of ERK activity in the cytosol and F-actin intensity at the 313 apical edge. Colors indicate the administration of different chemicals; red: $500 \mathrm{ng} \mathrm{mL}^{-1} \mathrm{FGF} 1$, $314 \mathrm{n}=1204, \mathrm{~N}=4$, blue: $500 \mathrm{ng} \mathrm{mL}^{-1} \mathrm{FGF} 1$ and $1 \mu \mathrm{M}$ PD0325901 (MEKi), $\mathrm{n}=687, \mathrm{~N}=3$, green: $315100 \mathrm{ng} \mathrm{mL}^{-1} \mathrm{FGF} 7, \mathrm{n}=787, \mathrm{~N}=3$. 


\section{5. ERK activation flattens epithelial tissues via apical actin polymerization}

318 How does the ERK-mediated actin polymerization at the apical side of cells result in

319 morphological changes in monolayer tissues? To answer this, we used a simple geometrical

320 model of a two-dimensional monolayer tissue, composed of cells with identical morphologies.

321 In this model, individual cells are depicted by tetragons, the edges of which each correspond

322 to the apical, basal, and lateral sides of cells. Then, the sheet curvature $\kappa$ can be represented

323 as a function of the length of these edges; especially, a change in the curvature with respect to

324 the apical edge length $a$ can be derived as $\partial \kappa / \partial a<0$. Thus, the sheet curvature decreases

325 with an expansion in the apical edge (see Materials and Methods IIb). This theoretical analysis

326 predicts that ERK activation would decrease the tissue curvature via actin polymerization at

327 the apical edges.

328 To confirm this prediction, we examined the change in epithelial tissue curvature in 329 response to acute ERK activation. For this purpose, an isolated lung epithelium was incubated 330 in the culture medium without any growth factors. Then, FGF1 was added into the medium to 331 simulate ERK. Acute ERK activation exclusively promoted F-actin accumulation at the apical 332 side of cells in epithelial distal tips; this was followed by the flattening of the epithelial sheet 333 (Figure 5A-D, Movie 7). Moreover, the simultaneous administration of FGF1 and PD0325901 334 resulted in neither F-actin accumulation nor tip flattening; this was also observed for PBS 335 control treatment (Figure 5B-D). These results indicate that ERK activation flattens the 336 epithelial tissues in the tips via actin polymerization at the apical sides of cells. 
A
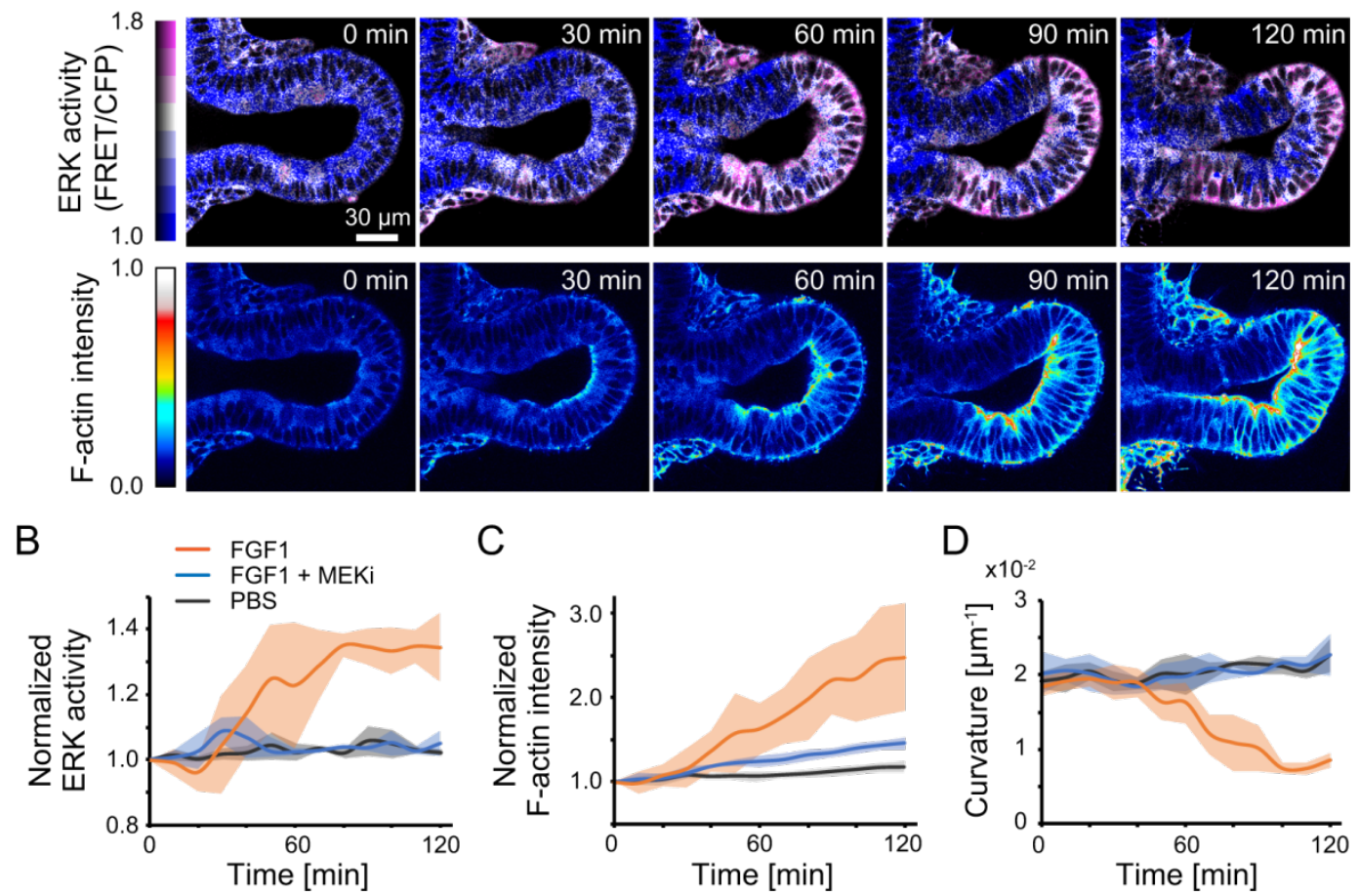

340 Figure 5 Simultaneous imaging of FGF-triggered ERK activation, actin polymerization, 341 and tissue flattening

342 (A) Time-lapse images of ERK activity (upper) and F-actin intensity (lower). SiR-Actin was 343 pretreated for F-actin visualization, 1 hour before the addition of FGF1. The time origin 344 indicates the timing of FGF1 administration. Scale bar, $30 \mu \mathrm{m}$.

345 (B-D) Normalized ERK activity in the tips (B), normalized F-actin intensity in the tips (C), 346 and tissue curvature at the tip apex (D) over time. Colors indicate different chemical treatments, 347 including with $500 \mathrm{ng} \mathrm{mL}^{-1} \mathrm{FGF} 1$ and $1 \mu \mathrm{M}$ PD0325901 (MEKi). The time origin indicates 348 the timing of chemical administration. Data represent mean and SD values. $\mathrm{N}=4$. 


\section{Feedback between ERK activity and tissue curvature explains repetitive branching}

351 To examine whether the curvature-dependent apical extension would be able to produce the 352 periodic branching pattern, we performed numerical analysis using vertex dynamics model, a 353 cell-based mechanical model ${ }^{30-32}$. The model represents epithelial cells as polygons with 354 vertices and edges, and the edges on the apical side actively deform via F-actin dynamics that 355 depend on the tissue-scale curvature and its decay (as described in Materials and Methods IIc). 356 We conducted numerical simulations with an initial shape and bud diameters obtained from the experiment (Figure 6A), and examined the impact of curvature on the change in apical edge length through a model parameter $\alpha$, using the standardized parameter set (Figure S4A). The apical edge extends/shrinks in accordance with the positive/negative values of $\alpha$ in a curvaturedependent manner (Figure 6B). Clearly, the curvature and cell height at the tip apex decreased with an increase in $\alpha$ at equilibrium (Figure 2B, S5B). Moreover, during curvature-dependent apical extension $(\alpha=1)$, the curvature decreases at the tip apex (arrowhead, Figure 2C), but increases at both sides over time (arrows, Figure 2C), indicating that the curvature-dependent apical extension can create new buds for terminal bifurcation. This does not happen in the opposite case $(\alpha=-1)$, suggesting that curvature-dependent apical constriction is not likely to drive repetitive branching, contradictory to a previous report ${ }^{33}$.

We then enabled cell proliferation to occur in the model system, in order to perform in silico experiments in virtual growing monolayer tissues. The simulation of the regime of curvature-dependent apical extension and cell proliferation clearly demonstrates the formation of repetitive branching patterns (Figure 6D, Movie 8). In contrast, the repetitive branching pattern is not generated in counterpart regimes, such as no regulation on the apical edge, and global apical extension as observed for the FGF7 treatment - apical extension happens in all cells, irrespective of curvature (Figure 6E). In these counterpart regimes, there are significant differences in both the averaged curvature, a measure of curvature averaged over the entire tissue, and the correlation coefficient between the curvature and monolayer thickness (Figure 6F, 6G). Moreover, the virtual experiments show that luminal pressure, which has been proposed to be a physical factor for lung morphogenesis ${ }^{34,35}$, is not essential for branching pattern formation; rather, it suppresses its constant large magnitude value (Figure S4C). These results support the fact that that the exclusive occurrence of apical edge extension in cells with high curvatures drives repetitive terminal bifurcation in the growing epithelial monolayer in a self-sustaining manner.

Furthermore, numerical investigations have clarified that the retention time of curvature memory in F-actin, i.e., the inverse of the F-actin decay rate $\gamma$, is crucial for generating the 
384 branching pattern (Figure $6 \mathrm{H}$, Movie 9-11). The averaged curvature becomes larger for a

385 longer curvature memory, and the correlation between the curvature and F-actin becomes

386 higher for a shorter curvature memory. Thus, there is an optimal range for the retention time

387 of the curvature memory in F-actin (Figure 6I). This result suggests the importance of the tissue

388 curvature hysteresis of cellular force generation via F-actin, for the emergence of repetitive

389 branch patterns.

390

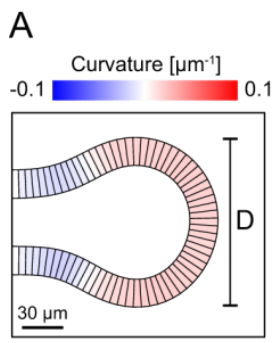

B

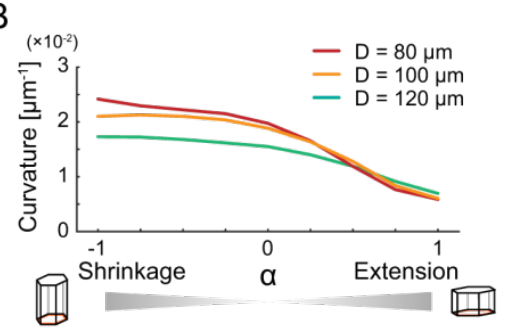

C
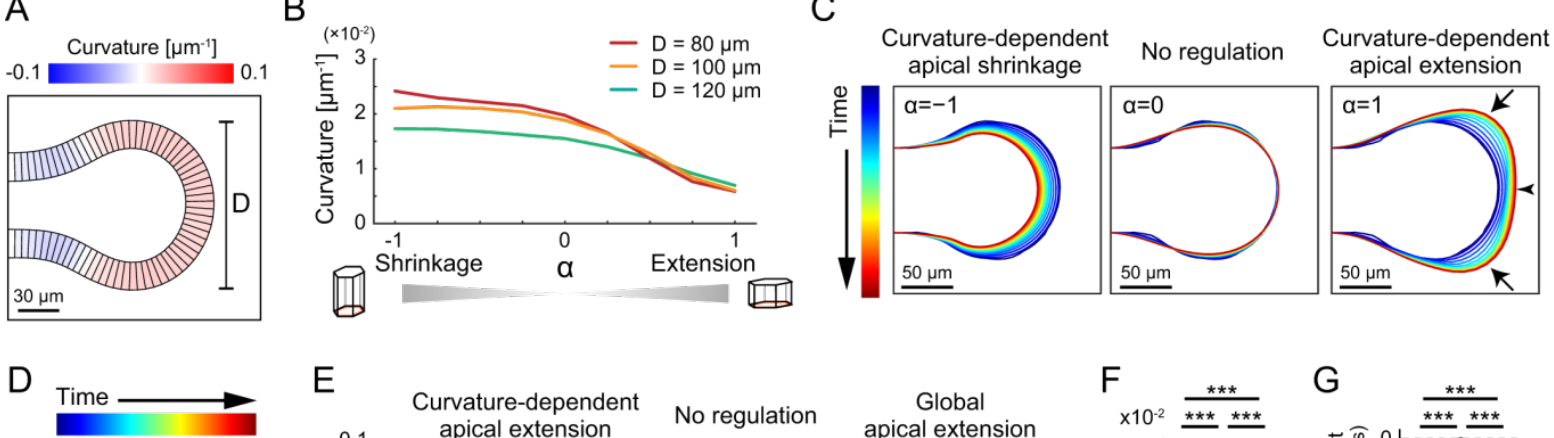

E
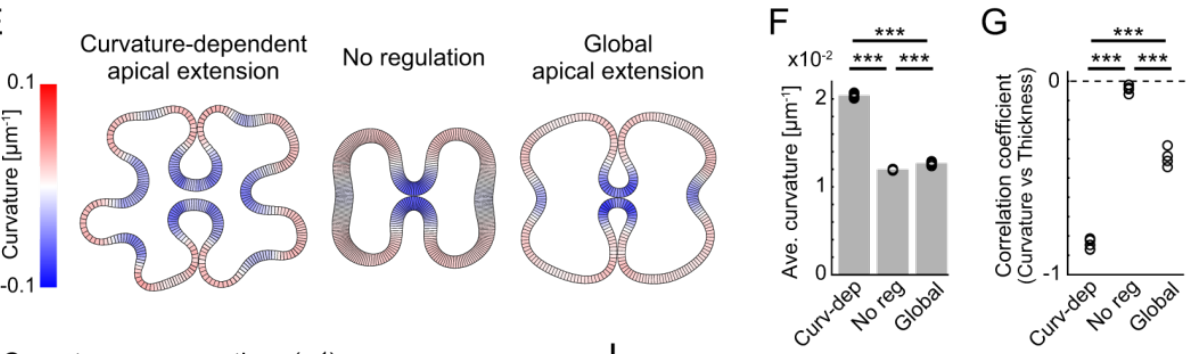

$\mathrm{H}$

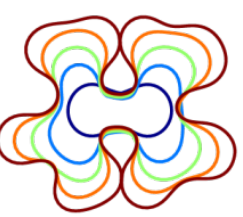

Curvature memory time $\left(\mathrm{Y}^{-1}\right) \quad$ Long

I
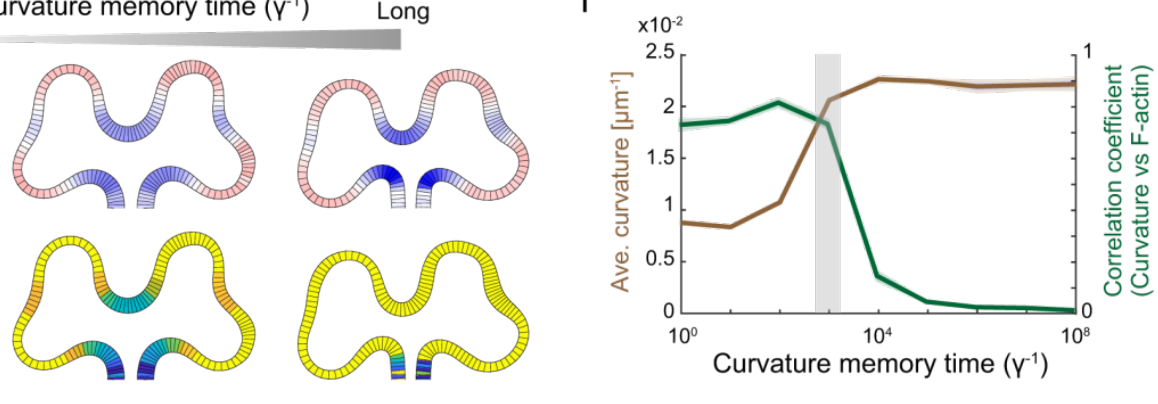

\section{Figure 6 Simulation and analysis of the mathematical model}

394 (A) Distal tip of a virtual epithelial monolayer tissue during the initial configuration of the simulation. Colors indicate the tissue curvature. D represents the diameter of the tip. Scale bar, $39630 \mu \mathrm{m}$.

397 (B) Tissue curvature at the tip apex over the parameter $\alpha$. Colors indicate the tip diameter.

398 (C) Time evolution of the basal sides of cells in different $\alpha$. Colors indicate the time periods in 399 simulations. The arrowhead indicates the tip apex and arrows indicate the regions with a 400 growing curvature. Scale bars, $50 \mu \mathrm{m}$.

401 (D) Time evolution of the basal sides of cells in the simulation with cell proliferation at $\alpha=1$.

402 (E) Generated morphology of epithelial monolayer tissues in different regimes. Curvature403 dependent apical extension: $\alpha=1, \beta=0$; no regulation: $\alpha=0, \beta=0$; global apical extension: $\alpha=0$, $404 \beta=0.2$. 
405 (F, G) Averaged curvature (F) and correlation coefficient between the tissue curvature and 406 tissue thickness $(\mathrm{G})$ for the 3 regulatory regimes. Bars represent mean values. $\mathrm{N}=5$. Welch's t407 test, $\mathrm{p}<0.001$.

408 (H) Spatial maps of the curvature (upper) and F-actin level (lower) for different curvature 409 memory time periods $\gamma^{-1}$. left: $\gamma=1$, middle: $\gamma=10^{-3}$, right: $\gamma=10^{-6}$.

410 (I) Tissue curvature (green) and correlation coefficient between the curvature and the F-actin 411 level. Data represent mean and SD values. $\mathrm{N}=5$. The gray shaded region indicates the region 412 in which both the values are high. 


\section{Discussion}

415 Here, we have demonstrated a regulatory mechanism by which epithelial monolayer cells 416 undergo repetitive branching morphogenesis through curvature sensing and ERK-mediated 417 mechanical force generation in a self-sustained manner. Our two-photon live imaging analysis 418 has shown that the ERK activity changes following a dynamic change in the tissue curvature, 419 and ERK activation occurs preferentially in curved epithelial tissues. Moreover, we found that 420 ERK-mediated actin polymerization contributes to the extension of the apical side of cells, and 421 demonstrated its importance for achieving a decrease in tissue curvature during terminal 422 bifurcation via mathematical model analyses. Thus, there is a closed negative feedback loop 423 between the tissue curvature and ERK activity across multiple scales in developing lung 424 epithelial tissues (Figure 7).

The results presented in this study provide a better understanding of lung epithelial branching, in conjunction with previously determined theory-based mechanisms, based on mechanical and chemical aspects. From a mechanical viewpoint, it has been proposed that the spatial patterning of the isolated lung epithelium arises from the proliferation-induced physical instability of the epithelial layer, known as mechanical buckling ${ }^{36}$, in various developing epithelial tissues $1,37,38$. This purely mechanical model may explain the formation of an initial branching pattern, under the assumption that the epithelial tissue acts as an elastic layer, but may require the physical conditions under which mechanical buckling occurs. However, our results have demonstrated that repetitive branching can be generated even at a range where proliferation-induced buckling did not occur (Figure 6E-G). We emphasize that the regime presented here is not mutually exclusive to buckling-induced patterning. Thus, ERK-mediated tissue curvature feedback results in a robust regulatory system underlying branching morphogenesis, together with the buckling regime. In addition, a simple reaction-diffusion model of substrate-depletion has been proposed for integrating chemical signal and tissue growth ${ }^{39,40}$; the model assumes that lung epithelial cells consume FGF ligands that have slow diffusion rates, and thereby predicts that a concentration gradient of FGF is generated in the extracellular matrix. However, live imaging analysis results have shown that no FGF gradient was formed in the Matrigel, while FGF was internalized in the epithelial cells through endocytosis (Figure S2). Other reaction-diffusion systems have focused on genetic regulatory networks that can achieve the localization of $F g f 10$ expression observed in vivo ${ }^{41-43}$. Although the spatial distribution of the chemoattractant is dispensable for epithelial branching ${ }^{18}$ (Figure 2D), the incorporation of such fundamental biochemical signals into our model would be useful for studying detailed systems for lung branching and patterning in vivo. 
Our findings highlight the curvature-sensitive ERK activation of lung epithelial cells and raise an important question about how cells sense the tissue curvature and transmit FGF-ERK signals. Because the ERK activity response occurs within $10 \mathrm{~min}$ after the perturbation in the tissue curvature (Figure 3B-E), the chemical feedbacks via gene expression, e.g., the interplay between the FGF and Sonic hedgehog expressed in the epithelium, are unlikely but rather via unknown protein-protein interactions should be involved in this process. We determined from the results (Figure $2 \mathrm{G}, 3 \mathrm{G}-\mathrm{H}$ ) that the phenomenon of curvature-sensitive ERK activation in lung epithelial tissues was attributable to a strain exhibiting a certain cell membrane or level of membrane tension, as shown previously for other tissues ${ }^{44-46}$. Moreover, the state of the membrane would trigger receptor-mediated endocytosis, as FGF internalization occurs exclusively in epithelial tissues with a positive curvature (Figure S2). Multiple intracellular events, including the endocytic trafficking of FGFR and molecular sensing of plasma membrane curvature, would be involved in this process ${ }^{47-49}$.

This study has focused on the core mechanism to minimally generate the self-sustained repetitive branching patterns in epithelium; however, there are other additional contributions that could achieve the robust tissue morphogenesis in vivo. For example, smooth muscle cells are not necessary for the initiation of cleft formation (Figure 2D) ${ }^{50}$, but should mechanically assist the cleft formation from the outside of an epithelium, realizing the stable terminal bifurcation ${ }^{27,50}$. Luminal fluid is another potentially important factor that can mechanically affect epithelial tissue deformation through shear stress and hydrostatic pressure ${ }^{51}$. It has been previously reported that the manipulation of luminal pressure in murine developing lungs under ex vivo culture conditions altered branch formation ${ }^{34}$. As oscillatory contractions of airway smooth muscles are likely to produce dynamic changes in the luminal pressure, it has been suggested that rhythmic luminal pressure would control the global synchronization of branching events in the murine lung ${ }^{35}$, although it does not contribute to the tissue flattening during the terminal bifurcation.

The ERK-mediated curvature feedback mechanism proposed here advances the concept of mechanochemical regulation of the epithelial monolayer ${ }^{32,44,52,53}$, in terms of taking the impact of tissue geometry into account. In various tissues, the epithelial cells collectively form their shape in the tissue scale and the constituent cells respond to tissue curvature at different levels, including the transcription and cell activity levels ${ }^{54-57}$. We believe that the proposed system works as a regulatory principle underlying the mechanisms of self-organized tissue morphogenesis. 


\section{Curvature-sensitive ERK activation}

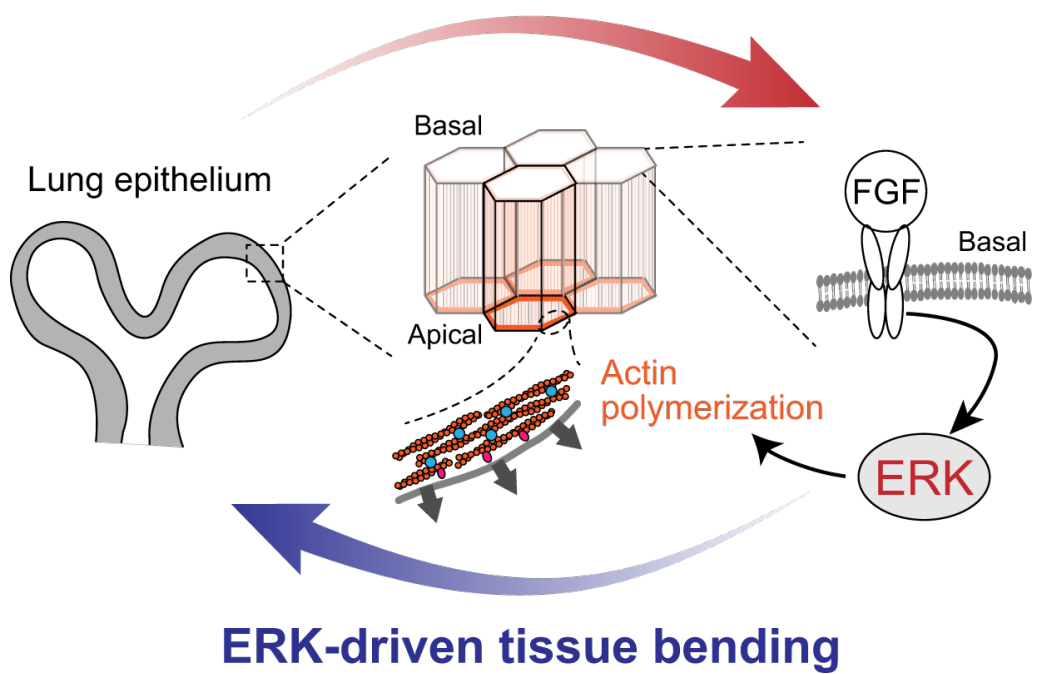

483

484 Figure 7 ERK-mediated curvature feedback regulates branching morphogenesis in 485 murine lung epithelial tissue

486 The epithelial cells activate ERK specifically in tissues with a positive curvature via reception 487 of extracellular FGF ligands (red arrow). ERK-mediated actin polymerization contributes to 488 the extension of the apical side of cells, which decreases the tissue curvature (blue arrow). Thus, 489 there is a closed negative feedback loop between tissue curvature and ERK activity across 490 multiple scales in developing epithelial lung tissues. 


\section{Acknowledgements}

493 This work was supported by the JST PRESTO grant JPMJPR1949, by the JSPS KAKENHI

494 19H00993, by the SPIRITS 2018 of Kyoto University, and by the Medical Research Support

495 Center of Kyoto University. We would like to thank Akane Kusumi for technical assistance,

496 Edouard Hannezo, Naoya Hino, Mamoru Ishii, and Takuya Yoshida for fruitful discussion.

497

498

\section{Author Contributions}

500 Conceptualization, T.H.; Methodology, T.H.; Software, T.H.; Validation, T.H.; Formal 501 analysis, T.H.; Investigation, T.H.; Resources, T.H., M.M.; Data curation, T.H.; Writing 502 original draft, T.H.; Writing - review \& editing, T.H., M.M.; Visualization, T.H.; Supervision, 503 T.H.; Project administration, T.H., M.M.; Funding acquisition, T.H., M.M.

\section{Competing interests}

507 The authors declare no competing interests. 


\section{References}

510 1. Hirashima, T. Pattern Formation of an Epithelial Tubule by Mechanical Instability

511 during Epididymal Development. Cell Rep. 9, 866-73 (2014).

512 2. Metzger, R. J., Klein, O. D., Martin, G. R. \& Krasnow, M. A. The branching 513 programme of mouse lung development. Nature 453, 745-750 (2008).

514 3. Hannezo, E. et al. A Unifying Theory of Branching Morphogenesis. Cell (2017).

515 doi:10.1016/j.cell.2017.08.026

516 4. Lu, P. \& Werb, Z. Patterning mechanisms of branched organs. Science (80-. ). 322, 517 1506-1509 (2008).

518 5. Cardoso, W. V. \& Lü, J. Regulation of early lung morphogenesis: questions, facts and controversies. Development 133, 1611-1624 (2006).

6. Warburton, D. et al. Lung organogenesis. in Current Topics in Developmental Biology

522 7. Herriges, M. \& Morrisey, E. E. Lung development: Orchestrating the generation and

8. Varner, V. D. \& Nelson, C. M. Cellular and physical mechanisms of branching morphogenesis. Development 141, 2750-9 (2014).

9. Ingber, D. E. Mechanical control of tissue morphogenesis during embryological

10. Anlaş, A. A. \& Nelson, C. M. Tissue mechanics regulates form, function, and

11. Ornitz, D. M. \& Itoh, N. The Fibroblast Growth Factor signaling pathway. Wiley Interdiscip. Rev. Dev. Biol. 4, 215-266 (2015).

12. Cardoso, W. V., Itoh, A., Nogawa, H., Mason, I. \& Brody, J. S. FGF-1 and FGF-7

13. Nogawa, H. \& Ito, T. Branching morphogenesis of embryonic mouse lung epithelium

14. Bellusci, S., Grindley, J., Emoto, H., Itoh, N. \& Hogan, B. L. M. Fibroblast Growth Factor 10 (FGF10) and branching morphogenesis in the embryonic mouse lung. Development (1997).

15. Min, H. et al. Fgf-10 is required for both limb and lung development and exhibits 
striking functional similarity to Drosophila branchless. Genes Dev. (1998). doi:10.1101/gad.12.20.3156

16. Sekine, K. et al. Fgf10 is essential for limb and lung formation. Nat. Genet. (1999). doi:10.1038/5096

17. Arman, E., Haffner-Krausz, R., Gorivodsky, M. \& Lonai, P. Fgfr2 is required for limb outgrowth and lung-branching morphogenesis. Proc. Natl. Acad. Sci. U. S. A. (1999). doi:10.1073/pnas.96.21.11895

18. Volckaert, T. et al. Localized Fgf10 expression is not required for lung branching morphogenesis but prevents differentiation of epithelial progenitors. Dev. (2013). doi:10.1242/dev.096560

19. Boucherat, O., Nadeau, V., Berube-Simard, F.-A., Charron, J. \& Jeannotte, L. Crucial

20. Yoshida, T., Matsuda, M. \& Hirashima, T. Incoherent Feedforward Regulation via Sox9 and ERK Underpins Mouse Tracheal Cartilage Development. Front. Cell Dev. Biol. 8, (2020).

21. Liu, Y. et al. Novel role for netrins in regulating epithelial behavior during lung branching morphogenesis. Curr. Biol. (2004). doi:10.1016/j.cub.2004.05.020

22. Jiang, K., Tang, Z., Li, J., Wang, F. \& Tang, N. Anxa4 mediated airway progenitor

23. Tang, N., Marshall, W. F., McMahon, M., Metzger, R. J. \& Martin, G. R. Control of cell migration promotes distal epithelial cell fate specification. Sci. Rep. (2018).

25. Komatsu, N. et al. Development of an optimized backbone of FRET biosensors for kinases and GTPases. Mol. Biol. Cell 22, 4647-56 (2011).

26. Harvey, C. D. et al. A genetically encoded fluorescent sensor of ERK activity. Proc. Natl. Acad. Sci. U. S. A. 105, 19264-9 (2008).

27. Kim, H. Y. et al. Localized Smooth Muscle Differentiation Is Essential for Epithelial (2015). doi:10.1016/j.devcel.2015.08.012

28. Yuan, T. et al. Temporospatial Expression of Fgfr1 and 2 During Lung Development, 
Homeostasis, and Regeneration. Front. Pharmacol. 11, (2020).

578

579

580

581

582

583

584

585

586

587

588

589

590

591

592

593

594

595

596

597

598

599

600

601

602

603

604

605

606

607

608

609

610

29. De Moerlooze, L. et al. An important role for the IIIb isoform of fibroblast growth factor receptor 2 (FGFR2) in mesenchymal-epithelial signalling during mouse organogenesis. Development 127, 483-492 (2000).

30. Fletcher, A. G., Osterfield, M., Baker, R. E. \& Shvartsman, S. Y. Vertex models of epithelial morphogenesis. Biophys. J. 106, 2291-2304 (2014).

31. Nagai, T. \& Honda, H. A dynamic cell model for the formation of epithelial tissues. Philos. Mag. B 81, 699-719 (2001).

32. Hirashima, T. \& Adachi, T. Polarized cellular mechano-response system for maintaining radial size in developing epithelial tubes. Development 146, dev181206 (2019).

33. Fumoto, K., Takigawa-Imamura, H., Sumiyama, K., Kaneiwa, T. \& Kikuchi, A. Modulation of apical constriction by Wnt signaling is required for lung epithelial shape transition. Development 144, 151-162 (2017).

34. Unbekandt, M. et al. Tracheal occlusion increases the rate of epithelial branching of embryonic mouse lung via the FGF10-FGFR2b-Sprouty2 pathway. Mech. Dev. 125, 314-324 (2008).

35. Nelson, C. M. et al. Microfluidic chest cavities reveal that transmural pressure controls the rate of lung development. Development (2017). doi:10.1242/dev.154823

36. Varner, V. D., Gleghorn, J. P., Miller, E., Radisky, D. C. \& Nelson, C. M. Mechanically patterning the embryonic airway epithelium. Proc. Natl. Acad. Sci. 112, 9230-9235 (2015).

37. Kourouklis, A. P. \& Nelson, C. M. Modeling branching morphogenesis using materials with programmable mechanical instabilities. Curr. Opin. Biomed. Eng. 6, 66-73 (2018).

38. Savin, T. et al. On the growth and form of the gut. Nature 476, 57-62 (2011).

39. Miura, T. \& Shiota, K. Depletion of FGF acts as a lateral inhibitory factor in lung branching morphogenesis in vitro. Mech. Dev. 116, 29-38 (2002).

40. Hartmann, D. \& Miura, T. Modelling in vitro lung branching morphogenesis during development. J. Theor. Biol. 242, 862-872 (2006).

41. Hirashima, T., Iwasa, Y. \& Morishita, Y. Mechanisms for split localization of Fgf10 expression in early lung development. Dev. Dyn. 238, 2813-2822 (2009).

42. Menshykau, D., Kraemer, C. \& Iber, D. Branch Mode Selection during Early Lung Development. PLoS Comput. Biol. 8, e1002377 (2012). 
611 43. Menshykau, D., Blanc, P., Unal, E., Sapin, V. \& Iber, D. An interplay of geometry and signaling enables robust lung branching morphogenesis. Development 141, 4526-4536

613 (2014).

614 44. Hino, N. et al. ERK-Mediated Mechanochemical Waves Direct Collective Cell Polarization. Dev. Cell 53, 646-660.e8 (2020).

45. Kinoshita, N. et al. Mechanical Stress Regulates Epithelial Tissue Integrity and Stiffness through the FGFR/Erk2 Signaling Pathway during Embryogenesis. Cell Rep. 30, 3875-3888.e3 (2020).

46. Sitarska, E. \& Diz-Muñoz, A. Pay attention to membrane tension: Mechanobiology of the cell surface. Curr. Opin. Cell Biol. 66, 11-18 (2020).

47. Francavilla, C. et al. Functional Proteomics Defines the Molecular Switch Underlying FGF Receptor Trafficking and Cellular Outputs. Mol. Cell 51, 707-722 (2013).

48. Belleudi, F. et al. Keratinocyte Growth Factor Receptor Ligands Target the Receptor to Different Intracellular Pathways. Traffic 8, 1854-1872 (2007).

49. Antonny, B. Mechanisms of Membrane Curvature Sensing. Annu. Rev. Biochem. 80, 101-123 (2011).

50. Young, R. E. et al. Airway Smooth Muscle Differentiation is Essential for Promoting

630 51. Chan, C. J. \& Hiiragi, T. Integration of luminal pressure and signalling in tissue selforganization. Development 147, dev181297 (2020). mechanochemical patterning and optimal migration in cell monolayers. Nat. Phys. 17, 267-274 (2021).

635 53. Ishii, M., Tateya, T., Matsuda, M. \& Hirashima, T. Retrograde ERK activation waves drive base-to-apex multicellular flow in murine cochlear duct morphogenesis. Elife 10, (2021).

54. Nikolaev, M. et al. Homeostatic mini-intestines through scaffold-guided organoid

640 55. Nelson, C. M., VanDuijn, M. M., Inman, J. L., Fletcher, D. A. \& Bissell, M. J. Tissue

643 56. Luciano, M. et al. Large-scale curvature sensing by epithelial monolayers depends on active cell mechanics and nuclear mechanoadaptation. bioRxiv (2020). 
doi:doi.org/10.1101/2020.07.04.187468

646

57. Hughes, A. J. et al. Engineered Tissue Folding by Mechanical Compaction of the

647 Mesenchyme. Dev. Cell 44, 165-178.e6 (2018).

648

58. Hirashima, T. \& Adachi, T. Procedures for the Quantification of Whole-Tissue Immunofluorescence Images Obtained at Single-Cell Resolution during Murine Tubular Organ Development. PLoS One 10, e0135343 (2015).

59. Hirashima, T. Mechanical Tissue Compression and Whole-mount Imaging at Single Cell Resolution for Developing Murine Epididymal Tubules. BIO-PROTOCOL 10,

654 60. Lukinavičius, G. et al. Fluorogenic probes for live-cell imaging of the cytoskeleton. Nat. Methods (2014). doi:10.1038/nmeth.2972

656

61. Milroy, L. G. et al. Selective chemical imaging of static actin in live cells. J. Am. Chem. Soc. (2012). doi:10.1021/ja211708z

62. Tran, A. P., Yan, S. \& Fang, Q. Improving model-based functional near-infrared spectroscopy analysis using mesh-based anatomical and light-transport models. Neurophotonics 7, 1 (2020).

661

63. Meyer, M., Desbrun, M., Schr, P. \& Barr, A. H. Discrete Differential-Geometry Operators for Triangulated 2-Manifolds. Vis. Math. III 113-134 (2003). doi:10.1016/j.cagd.2007.07.005

64. Hannezo, E., Prost, J. \& Joanny, J.-F. Theory of epithelial sheet morphology in three dimensions. Proc. Natl. Acad. Sci. 111, 27-32 (2014).

65. Wen, F. L., Wang, Y. C. \& Shibata, T. Epithelial Folding Driven by Apical or BasalLateral Modulation: Geometric Features, Mechanical Inference, and Boundary Effects.

669 66. Boucherat, O., Nadeau, V., Berube-Simard, F.-A., Charron, J. \& Jeannotte, L. Crucial 670 requirement of ERK/MAPK signaling in respiratory tract development. Development 142, 3197-3211 (2014). 
674 Materials and Methods

675 (I) Experiments

676 Animals

677 It has been previously reported that transgenic mice expressed hyBRET-ERK-NES, a FRET 678 biosensor ${ }^{24-26}$. hyBRET-ERK-NES is comprised of cyan fluorescent protein (CFP) and yellow 679 fluorescent protein (YFP), which act as the donor and acceptor of FRET, respectively. 680 Otherwise, we used ICR mice purchased from Japan SLC, Inc. We designated the midnight 681 preceding the discovery of the vaginal plug as embryonic day 0.0 (E0.0), and all mice were 682 sacrificed via cervical dislocation to minimize suffering. All animal experiments were 683 approved by the local ethical committee for animal experimentation (MedKyo 18086, 19090, 684 and 20081), and were performed in compliance with the guidelines for the care and use of 685 laboratory animals, at Kyoto University.

686

\section{Antibodies, small molecules, and recombinant proteins}

688 We used the following primary antibodies at a dilution ratio of 1:200 unless stated otherwise: 689 rat monoclonal anti-E-cadherin (Cell Signaling Technology, \#3195), rabbit polyclonal anti690 phospho-myosin light chain (pMLC) (Abcam, \#ab2480), Alexa Fluor 546-conjugated goat 691 anti-rat IgG (H+L) antibody (Thermo Fisher Scientific, \#A11081, 1:1000), Alexa Fluor 647692 conjugated goat anti-rabbit IgG $(\mathrm{H}+\mathrm{L})$ antibody (Abcam, \#ab150079, or Thermo Fisher 693 Scientific, \#A21247, 1:1000), Blebbistatin (Merck Millipore, \#203391), CK-666 (Sigma694 Aldrich, \#SML0006), Cytochalasin D (Merck Millipore, \#250255), FGF1 (R\&D Systems, 695 \#232-FA), FGF7 (R\&D Systems, \#251-KG), Jasplakinolide (Abcam, \#ab141409), Latrunculin 696 A (Cayman Chemical, \#10010630), Nocodazole (Merck Millipore, \#487928), PD0325901 697 (FUJIFILM Wako Pure Chemical Corporation, \#162-25291), SMIFH2 (Sigma-Aldrich, 698 \#S4826), Y-27632 (Merck Millipore, \#SCM075).

699

\section{$700 \quad$ Ex vivo culture}

701 Dissected lung lobes were placed onto a hydrophilic polytetrafluoroethylene organ culture 702 insert with a pore size of $0.4 \mu \mathrm{m}$ (Merck Millipore, \#PICM01250), which was preset in a 35 $703 \mathrm{~mm}$ petri dish filled with $800 \mu \mathrm{L}$ of culture medium. Samples were cultured in an air-liquid 704 interface at $37^{\circ} \mathrm{C}$ under $5 \% \mathrm{CO}_{2}$ conditions. To culture isolated epithelial tissues, dissected 705 lobes were digested with $10 \mathrm{PU} / \mathrm{mL}$ of Dispase I (FUJIFILM Wako Pure Chemical Corporation, 706 \#386-02271) in phosphate buffered saline (PBS) for $15 \mathrm{~min}$ on ice, and immersed into fetal 707 bovine serum (FBS) and kept on ice for $5 \mathrm{~min}$, to stop the protease-mediated reaction. Then, 
708

709

710

711

712

713

714

\section{5}

716

717

718

719

720

721

722

723

724

725

726

727

728

729

730

731

732

733

734

735

736

737

738

739

740

741

samples were washed in PBS twice on ice, and the distal tips of epithelial tissues were manually excised using fine needles. Isolated epithelial tissues were cultured within growth factorreduced Matrigel (Corning, \#356231) and covered with $500 \mu \mathrm{L}$ of culture medium added to each well of glass bottom dishes (Greiner, \#627870) at $37^{\circ} \mathrm{C}$ under $5 \% \mathrm{CO}_{2}$ conditions. We used FluoroBrite DMEM Media (Thermo Fischer Scientific, \#A1896701) containing 1\% GlutaMAX (Thermo Fischer Scientific, \#35050061) and 0.5\% FBS as the culture medium.

\section{Whole-tissue immune-fluorescence staining}

Staining and optical clearing of dissected lung were performed according to previous studies ${ }^{58,59}$. Briefly, samples were fixed with $4 \%$ paraformaldehyde (PFA) in PBS overnight at $4{ }^{\circ} \mathrm{C}$. For pMLC staining, samples were fixed with $2 \%$ trichloroacetic acid in PBS containing a 1\% phosphatase inhibitor cocktail (1:100, Nacalai Tesque, \#07575-51) for $15 \mathrm{~min}$ at $4^{\circ} \mathrm{C}$. Then, samples were blocked by incubation in 10\% normal goat serum (Abcam, \#ab156046) diluted in $0.1 \%$ Triton $\mathrm{X}-100 / \mathrm{PBS}$ (PBT) for $3 \mathrm{~h}$ at $37^{\circ} \mathrm{C}$. Samples were treated with primary antibodies overnight at $4{ }^{\circ} \mathrm{C}$, washed in $0.1 \%$ PBT, and subsequently treated with secondary antibodies conjugated to either Alexa Fluor 546 or Alexa Fluor 647 overnight at $4{ }^{\circ} \mathrm{C}$. For nuclear counterstaining, we used Hoechst33342 (5 $\mu \mathrm{g} / \mathrm{ml}$, Dojindo Molecular Technologies, \#H342) or DAPI (Dojindo Molecular Technologies, \#D523-10, 1:200). The samples were mounted with $10 \mu \mathrm{L}$ of $1 \%$ agarose gel onto a glass-based dish (Greiner Bio-One, \#627871) for obtaining stable images. Then, samples were immersed with CUBIC-R+ (Tokyo Chemical Industry Co., \# T3741) solution for 1 hour at $37^{\circ} \mathrm{C}$ or BABB solution (benzyl-alcohol and benzyl-benzoate, $1: 2$ ) for $30 \mathrm{~min}$ at $23^{\circ} \mathrm{C}$.

\section{F-actin and tubulin labeling}

To visualize F-actin and microtubules, we used SiR-Actin and SiR-tubulin, respectively ${ }^{60,61}$, according to the manufacturer's instructions (Cytoskeleton, Inc. \#CY-SC001 and \#CY-SC002). We added $1 \mu \mathrm{M}$ of far-red fluorogenic probes to the culture media, 1 hour before imaging. Although SiR-Actin was derived from Jasplakinolide, which activated ERK in isolated lung epithelial tissues in our experiments, ERK activation did not occur after treatment with SiRActin in isolated lung epithelial tissues.

\section{Fluorescence labeling of FGF}

To visualizes internalized FGFs, we used an amine-reactive pH-sensitive pHrodo iFL Red STP ester dye, according to the manufacturer's instructions (Thermo Fischer Scientific, \#P36014). 
742 For the imaging of FGF1, regardless of its localization inside or outside cells, we used the

743 Alexa Fluor 488 Microscale Protein Labeling Kit, according to the manufacturer's instructions

744 (Thermo Fischer Scientific, \#A30006).

\section{Microscopy}

747 For live imaging, we used the incubator-integrated multiphoton fluorescence microscope 748 system (LCV-MPE, Olympus) with a $25 \times$ water-immersion lens (NA=1.05, WD=2 mm, 749 XLPLN25XWMP2, Olympus) or an inverted microscope (FV1200MPE-IX83, Olympus) with 750 a $30 \times$ silicone-immersion lens ( $\mathrm{NA}=1.05, \mathrm{WD}=0.8 \mathrm{~mm}$, UPLSAPO30XS, Olympus). The 751 excitation wavelengths were set to $840 \mathrm{~nm}$ for the CFP of the ERK FRET biosensor, $930 \mathrm{~nm}$ 752 for Alexa Fluor 488-labeled FGF1, and $1040 \mathrm{~nm}$ for the pHrodo iFL Red-labeled FGFs and 753 the SiR probes (InSight DeepSee, Spectra-Physics). We used RDM690 IR cut filters (Olympus), 754 and the DM505 and DM570 dichroic mirrors. We used BA460-500, BA495-540, BA520-560, 755 BA575-630, and BA647/57 emission filters (Olympus) for CFP, Alexa Fluor 488, YFP or 756 FRET, iFL Red probes, and SiR probes, respectively. For fixed samples, images were obtained 757 using the Leica TCS SP8 confocal laser scanning platform equipped with the Leica HyD hybrid detector, using the $20 \times$ objective lens $(\mathrm{NA}=0.75, \mathrm{WD}=680 \mu \mathrm{m}, \mathrm{HC}$ PL APO CS2, Leica) and $40 \times$ objective lens (NA $=1.3, \mathrm{WD}=240 \mu \mathrm{m}, \mathrm{HC}$ PL APO CS2, Leica).

\section{FRET image analysis}

762 The median filter of a $3 \times 3$ window was processed to remove shot noise, and the background 763 signal was subtracted from each FRET and CFP channel. Then, the ratio of FRET intensity to 764 CFP intensity was calculated using a custom-made MATLAB (MathWorks) script. In the scale 765 bar, the color represents the FRET/CFP ratio and brightness represents the fluorescence 766 intensity of the FRET channel.

\section{Three-dimensional mapping of different quantities}

769 To determine the correlation between different quantities, such as ERK activity, F-actin signal, 770 tissue curvature, and tissue thickness (Figure S1A), we obtained 3D stacked images of lung epithelial tissues with 1- $\mu \mathrm{m}$-long z-intervals, and manually traced either the basal or apical side

772 of the cells. Next, we generated triangle meshes on the traced surface using "Iso2Mesh," a free 773 mesh generation toolbox ${ }^{62}$. Then, we calculated a discrete Gaussian curvature and a discrete 774 curvature on each node of generated meshes using a custom-made code according to the 775 definition proposed earlier ${ }^{63}$. Finally, we obtained all values except those for the curvatures 
by averaging values around the nodes, and mapped them at each node. All processing was performed using MATLAB.

778

\section{Curvature measurement in 2D}

780 We used a spline curve-based method described elsewhere ${ }^{53}$. Briefly, we manually traced the 781 basal side of epithelial cells from images, and determined discrete sampling points $\left(x_{i}, y_{i}\right)$ 782 along traced curves at regular intervals. Upon fitting the 4 sampling points from $i-1$ to $i+2$ with 783 a cubic spline function, the function $S_{i}$ at an interval $\left[x_{i}, x_{i+1}\right]$ was denoted as $S_{i}(x)=$ $784 a_{i}\left(x-x_{i}\right)^{3}+b_{i}\left(x-x_{i}\right)^{2}+c_{i}\left(x-x_{i}\right)+d_{i}$. Due to the definition of the curvature using the 785 formula $\kappa(x)=S^{\prime \prime}\left(1+S^{\prime 2}\right)^{-3 / 2}$, the curvature from the spline function was calculated as 786 follows:

$$
\kappa_{i}(x)=\frac{6 a\left(x-x_{i}\right)+2 b}{\left(1+\left\{3 a\left(x-x_{i}\right)^{2}+2 b\left(x-x_{i}\right)+c\right\}^{2}\right)^{3 / 2}} .
$$

789

790 The convex/concave surface of the lumen was assigned a positive/negative value, namely $\kappa$. We defined tissue thickness as the length from the apical to the basal edge, which is vertical to 792 the traced curve at the sampling points.

\section{Mechanical strain assay}

795 We used a mechanical device (STREX, \#STB-10) with a polydimethylsiloxane (PDMS) 796 chamber (STREX, \#STB-CH-04). To compress isolated epithelium, we placed isolated lung epithelial tissues in a 50\% stretched state in the PDMS chamber, and added $1 \mathrm{~mL}$ of growth factor-reduced Matrigel (Corning, \#356231), and performed gelation for $15 \mathrm{~min}$ at $37^{\circ} \mathrm{C}$. Then, we added $2 \mathrm{~mL}$ of the culture medium supplemented with $500 \mathrm{ng} / \mathrm{mL}$ FGF1 into the chamber.

800 We conducted experiments involving epithelial tissues under 2D culture conditions in the

801 following manner. To obtain cell suspensions from primary tissues, lung tissues obtained via 802 dissection at E12.5 were treated with $1 \mathrm{mg} / \mathrm{mL}$ Collagenase/Dispase (Sigma-Aldrich, $803 \# 10269638001)$ in $\mathrm{PBS}$ for $50 \mathrm{~min}$ at $37^{\circ} \mathrm{C}$ while agitating the solution. Lung cells were then 804 dispersed into the solution by gentle pipetting, and the suspended solution was filtered using a $80540 \mu \mathrm{m}$ pore size mesh (Corning, \#352340). After centrifugation at $200 \mathrm{G}$ for $5 \mathrm{~min}$ at $25^{\circ} \mathrm{C}$, the 806 cell pellet was resuspended in a culture medium containing $10 \mu \mathrm{M} \mathrm{Y}-27632$ and 10\% 807 KnockOut Serum Replacement SR (Thermo Fischer Scientific, \# 10828010) and the solution 808 was placed in a PDMS chamber pretreated with a collagen coating (Cellmatrix Type I-C, Nitta 
809 Gelatin, \#631-00771). After 2 days, the culture medium was exchanged with a medium not 810 containing Y-27632 and KSR, 1 hour before imaging. Notably, the PDMS chamber gradually

811 droops by becoming attached to the silicone oil used for objective lenses, because of which the

812 focus has to be adjusted manually every time an image needs to be obtained.

813

\section{Statistical hypothesis testing}

815 The number of cells or regions of interest analyzed (n) and the number of biological replicates

816 (N) are indicated in the figure legends. No particular statistical method was used to

817 predetermine the sample size. A minimum of $\mathrm{N}=3$ independent experiments were performed,

818 based on previous studies in the field. No inclusion/exclusion criteria were used and all

819 analyzed samples were included in the analysis. No randomization was performed. Statistical

820 tests, sample sizes, test statistics, and $P$-values have been described in the main text. We

821 considered P-values $<0.05$ to be statistically significant in two-tailed tests, and classified them

822 into the following 4 categories: $*(\mathrm{p}<0.05),{ }^{* *}(\mathrm{p}<0.01),{ }^{* * *}(\mathrm{p}<0.001)$, and n.s. (not significant,

823 i.e., $\mathrm{p} \geq 0.05)$.

824

825 Software

826 We used MATLAB (MathWorks) and Image J (National Institute of Health) software for 827 digital image processing. For graphics, MATLAB (MathWorks), Imaris (Bitplane), and Image $828 \mathrm{~J}$ (National Institute of Health) were used. MATLAB (MathWorks) was used for statistical 829 analysis.

830

831 Graph

832 In the boxplot, the central mark indicates the median, and the bottom and top edges of the box

833 indicate the $25^{\text {th }}$ and $75^{\text {th }}$ percentiles, respectively. The whiskers extend to the most extreme 834 data points not considered to be outliers, and the outliers are plotted individually using the ' + ' 835 symbol. All the graphs were drawn using MATLAB.

838 (II) Model analysis

\section{9 (IIa) Apical length vs cell height at equilibrium}

840 Here, we consider cells in a flat epithelial sheet to be identical, and assume that the basal edge

841 length is almost the same as the apical edge length for the simplicity of analysis. Then, potential 842 energy can be defined as follows: 


$$
U_{\text {cell }}=\frac{k_{A}}{2}\left(A-A^{*}\right)^{2}+\frac{k_{a}}{2}\left(a-a^{*}\right)^{2}+k_{l} l
$$

845

846 The first term represents the cell size constraint and its coefficient $k_{A}$, current cell area $A$, and

847 the target cell area $A^{*}$. The second and third terms each represent the regulation of cell edge

848 length at the apical and lateral side of cells $a$ and $l$ with controlling parameters $k_{a}$ and $k_{l}$.

849 We set the second term as a function that converges to the variable target apical length $a^{*}$, as

850 explained later. Since the cell size was maintained even after a change in cell shape, caused by

851 the inhibitor assay, we set $A=A^{*}$. Then, upon considering that $A=r h$, we can redefine

852 equation 1 as follows:

853

$$
U_{\text {cell }}^{*}=\frac{k_{a}}{2}\left(a-a^{*}\right)^{2}+k_{l} \frac{A^{*}}{a}
$$

856 Now, we have two variables, $a$ and $a^{*}$, and obtained equilibrium values at the minimum potential energy value, i.e., $a_{e q}=\left(a \mid \partial U_{c e l l}^{*} / \partial a=0\right)$ and $a_{e q}^{*}=\left(a^{*} \mid \partial U_{c e l l}^{*} / \partial a^{*}=0\right)$ each, as $a_{e q}=\sqrt{A^{*} k_{l} / k_{b}}$ and $a_{e q}^{*}=a_{e q}$. From these equations, it was noted that $a_{e q}^{*} \sim l_{e q}^{-1}$, which indicates the existence of an inverse relationship between the apical length and cell height at equilibrium. The analysis in this section is based on that of a previous study ${ }^{64}$.

861

862

\section{(IIb) Apical length vs epithelial sheet curvature}

863 The analysis in this section is based on that of a previous study ${ }^{65}$. Here, we considered the

$$
\kappa=\frac{2}{a+b}\left(\pi-2 \cos ^{-1} \frac{b-a}{2 l}\right)
$$

$$
\frac{\partial \kappa}{\partial a}=-\frac{2}{l(a+b) \sqrt{1-\{(a-b) / 4 l\}^{2}}}-\frac{2}{(a+b)^{2}}\left(\pi-2 \cos ^{-1} \frac{b-a}{2 l}\right)<0 .
$$


874 This indicates that the sheet curvature becomes small with an increase in the apical edge length.

875

876

\section{(IIc) Mathematical modeling for multicellular dynamics}

878 We modeled an epithelial monolayer using the vertex model framework ${ }^{30-32}$. In our model, a 879 single cell is represented as a polygon with four vertices and edges, which are shared by 880 neighboring cells. The dynamics of vertex position $\mathbf{r}_{i}$, where $i$ is an index of the vertex, obeys the equation of motion, based on the principle of least potential energy $U$, with a drag coefficient $\eta$ and luminal pressure $p$ as follows:

$$
\eta \dot{\mathbf{r}}_{i}=-\nabla_{i} U+f(p)
$$

To determine the potential energy in the first term, we defined a minimal expression:

887

888

$$
U=\sum_{j}\left\{\frac{k_{A}}{2}\left(A_{j}-A_{j}^{*}\right)^{2}+\frac{k_{a}}{2}\left(a_{j}-a_{j}^{*}\right)^{2}+k_{l} l_{j}\right\}+\sum_{i} \frac{k_{\theta}}{2}\left\{\left(\theta_{i_{+}}-\pi / 2\right)^{2}+\left(\theta_{i_{-}}-\pi / 2\right)^{2}\right\}
$$

890 The first term represents the cell size constraint with its coefficient $k_{A}$, current cell area $A_{j}$, and the target cell area $A_{j}^{*}$. The second and third terms each represent the regulation of the cell edge length at the apical and the lateral sides of cells $a_{j}$ and $l_{j}$, with controlling parameters

$893 k_{a}$ and $k_{l}$. As the apical edge is controlled by the ERK activation level, we set the second term 894 as a function that converges to the variable target apical length $a_{j}^{*}$; this has been explained 895 later. The fourth and fifth terms represent the bending energy of cells at the apical and the basal 896 sides, attributed to each vertex $i ; k_{\theta}$ denotes the bending rigidity, and $\theta_{i_{+}}$and $\theta_{i_{-}}$are angles 897 at the vertex $i$ across the cell edge. on the apical edge of epithelial cells. The function $f$ was defined as

$$
f(p)=p \mathbf{n}_{i}
$$

902

903 where $p$ is strength of pressure, and $\mathbf{n}_{i}$ is the normal vector at the apical vertex $i$. Note that this 904 function is effective only at vertices in the apical sides of cells. 
To implement cell proliferation, we ensured that each cell was set with the cell timer $\tau_{j}$, and all cells had the same cell cycle length $\tau_{d i v}$. We assumed that cell cycle progression depended on the curvature; i.e., the cell cycle progresses when the cell curvature is not less than zero $\left(\kappa_{j} \geq 0\right)$, and the cell cycle pauses when the cell curvature is less than zero $\left(\kappa_{j}<0\right)$, according to the previous report ${ }^{36}$. This is also attributable to the fact that cell proliferation in the developing murine lung requires ERK activation, which can be observed in the distal tip 23,66. Cell division occurs and cells with almost equal sizes are produced when the cell timer reaches the cell cycle length, $\tau_{j}=\tau_{d i v}$. Once cell division occurs, the $\tau_{j}$ value in one of the daughter cells resets to zero and that in another is set at a value chosen stochastically from 0 to

$9140.05 \tau_{\text {div }}$ with a uniform distribution, to avoid perfect synchronization between neighboring 915 cells.

916 In our model, the target apical edge length depends on the cytoskeletal level $c$, observed 917 with a level of F-actin accumulation, as follows:

$$
a_{j}^{*}=\zeta g\left(c_{j}\right)+a_{0}
$$

920

$$
g\left(c_{j}\right)=\left\{\begin{array}{cl}
-1, & c_{j} \leq-1 \\
c_{j}, & -1<c_{j}<1 \\
1, & 1 \leq c_{j}
\end{array}\right.
$$

922

923 Here, $\zeta$ denotes the maximum length after edge extension, and $a_{0}$ is the baseline for the apical 924 edge length. The saturation function $g$ represents the physical limit for the change in edge 925 length. We modeled the cytoskeletal level $c$ as follows:

$$
\dot{c}_{J}=\alpha \tanh \left(\lambda \kappa_{j}\right) H\left(\kappa_{j}\right)+\beta-\gamma c_{j}
$$

929 The first term $\dot{c_{j}}$ represents the curvature-dependent contribution of FGF-ERK, along with the 930 magnitude parameter $\alpha$ and the sensitivity parameter $\lambda$, to the curvature $\kappa . H$ is the step 931 function of the curvature, i.e., $H=1$ for $\kappa \geq 0$ and $H=0$ for $\kappa<0$. The curvature $\kappa_{j}$ at cell $j$ 932 is calculated using the spline function-based method described above. As the cytoskeleton level 933 controls the apical target length observed with an increase in the monotonic function, as shown 934 in Equation 9, curvature-dependent apical extension/shrinkage is represented as a 935 positive/negative $\alpha$ value. The second term represents the curvature-independent contribution 
936 of FGF-ERK, along with the parameter $\beta$. The third term represents the decay of cytoskeletons,

937 including F-actin depolymerization and severing. The rate $\gamma$ controls the characteristic decay

938 time; the inverse value of $\gamma$ denotes the retention time for cell curvature memory. The level of

939 curvature-dependent FGF-ERK activation was determined in Equation 10 because of its rapid

940 response.

\section{2 (IId) Numerical simulations}

943 We considered a manually traced line along the basal sides of cells in the image shown in

944 Figure 1 as the initial shape for the simulation, when the diameter of the distal tip $D$ was in the

945 measured range. Upon an analysis of the tissue morphology, the number of cells was

946 determined according to the measured cell size, i.e., the average cell width and cell height,

947 which were $5 \mu \mathrm{m}$ and $20 \mu \mathrm{m}$, respectively. The boundary in the proximal side was fixed in the

948 simulations shown in Figures 6B and 6C. For the simulations in Figure 6D-I, we used the

949 symmetrical image created based on the Figure 6A. Ordinary differential equations were

950 numerically solved using the forward Euler method, with a time step of 0.01, using MATLAB

951 (MathWorks). The simulations were terminated when the number of cells was more than 300.

952 Standard parameters were set as follows: $\eta=1, k_{A}=0.01, k_{a}=1, k_{l}=0.2, k_{\theta}=$

$95320, \tau_{\text {div }}=300, \alpha=1, \beta=0, \gamma=0.001, \zeta=3 \mu m, \lambda=100, p=0.05$, and $D=$

$95470 \mu \mathrm{m}$, unless otherwise noted. The $k_{A}, k_{a}, k_{l}, k_{\theta}, \lambda$, and $p$ values were determined by

955 numerical analyses using several criteria, under which the simulated curvature imitates the

956 experimental observations and became insensitive to the change in the values, as shown in the

957 supplementary figure S4. The maximum length of edge extension, i.e., the $\zeta$ value, was

958 determined from our measurements. $A_{j}^{*}$ and $a_{0}$ values were set according to the initial

959 conditions, e.g., $A_{j}^{*}=93 \mu \mathrm{m}$ and $a_{0}=4 \mu \mathrm{m}$ when $D=70 \mu \mathrm{m}$. 


\section{Supplementary figure legends}

962

963 Figure S1 3D mapping of ERK activity and tissue geometry

964 (A) Mapping procedure for linking different quantities, including the ERK activity, epithelial 965 sheet curvature, and sheet thickness. Details of the procedure are described in the Materials 966 and Methods section. (B) The relationship between the ERK activity and discrete mean curvature. $\rho=0.61 \pm 0.04$, where $\rho$ is Spearman's rank correlation coefficient. Data represent mean and SD values. $n=427, \mathrm{~N}=3$.

969

\section{Figure S2 FGF1 endocytosis from the basal side of an isolated lung epithelium}

971 (A) Time-lapse images of the bright field (upper), FGF1-pHrodo (middle), and FGF1972 Alexa488 (lower). Red and blue circles in the bright field images indicate the tip apex and the 973 cleft bottom, respectively. The time origin indicates the time of administration of labeled FGF1.

974 FGF1 is distributed only in the Matrigel, not in the lumen, indicating that FGF1 internalization975 based endocytosis occurs from the basal side of the epithelium. Notably, FGF1 distribution is 976 almost homogeneous in the Matrigel (lower), indicating that no clear gradient was formed near 977 the epithelium, even after ligand internalization. Scale bar, $50 \mu \mathrm{m}$.

978 (B) FGF1-pHrodo intensity at the tip apex (red) and the cleft bottom (blue) over time. The 979 response time of FGF1-pHrodo at the tip apex is $86 \mathrm{~min} . \mathrm{N}=3$.

980 (C) FGF1-Alexa488 intensity in the Matrigel over time. The response time of FGF1-Alexa488 981 in the Matrigel is $26 \mathrm{~min} . \mathrm{N}=3$.

983 Figure S3 Morphological changes owing to chemical perturbations and cytoskeletal 984 architecture

985 (A) Morphological change in isolated epithelial cells after treatment with $1 \mu \mathrm{M}$ PD0325901. 986 Colors indicate the ERK activity level. Scale bar, $30 \mu \mathrm{m}$.

987 (B, C) Isolated lung epithelium stained with SiR-Actin (B) and with SiR-Tubulin (C). Scale 988 bar, $30 \mu \mathrm{m}$.

989 (D) Relative change in cell height after treatment with cytoskeletal inhibitors, such as $1 \mu \mathrm{M}$ 990 Latrunculin A, $1 \mu \mathrm{M}$ Nocodazole, and $30 \mu \mathrm{M}$ Blebbistatin. $\mathrm{N} \geq 3$ for each treatment.

991 (E-E") Immunofluorescence images of cells interacting with anti-E-cadherin (blue), anti992 phospho myosin light chain (pMLC, magenta) and the Hoechst (white) nuclear counterstain.

993 Notably, the pMLC signal is significantly weaker at the tip of the epithelium than in smooth 994 muscle cells around the stalk. In the magnified view (E"), the pMLC signal is clear at the 
995 microtubule organizing centers (arrowhead), and is localized at the apical side of cells despite

996 its weak intensity. Scale bars, $100 \mu \mathrm{m}$ (E, E') and $20 \mu \mathrm{m}\left(\mathrm{E}^{\prime \prime}\right)$.

997

\section{Figure S4 Simulation of the mathematical model}

999 (A) Parameter dependency of the monolayer curvature in the simulation.

1000 (B) Relative cell height, normalized by one at $\alpha=0$, as a function of $\alpha$. Representative

1001 morphologies of the virtual monolayer sheet are shown in the upper image.

1002 (C) Tissue curvature as a function of luminal pressure $p$. Representative morphologies of 1003 virtual monolayer tissues are shown in the upper image. $\mathrm{N}=5$. 
Movie legends

1006

\section{Movie 1 Volumetric imaging of ERK activity in the murine lung}

1008 The 3D ERK activity map of a murine lung dissected at E12.5. Colors represent the ERK

1009 activity and the color scale corresponds to that in Figure 1B. Scale bar, $50 \mu \mathrm{m}$.

1010

1011 Movie 2 Endocytosis of FGF1 in the isolated lung epithelium

1012 Time-lapse video of bright field (left), FGF1-Alexa488 (middle), and FGF1-pHrodo (right) in

1013 the isolated lung epithelium embedded within the Matrigel. The color scales correspond to

1014 those in Figure S2A. The time origin indicates the time of administration. Scale bars, $50 \mu \mathrm{m}$.

1015

1016

\section{Movie 3 Dynamics of ERK activity in the growing isolated lung epithelium}

1017 Time-lapse video of bright field (left) and ERK activity (right) during the terminal bifurcation 1018 of an isolated lung epithelium. The color scale corresponds to that in Figure 2D. Scale bars, 30 $1019 \mu \mathrm{m}$.

1020

1021

Movie 4 ERK activity response in the isolated lung epithelium to compression parallel to the distal-proximal axis

1023

Time-lapse video of ERK activity at the tip of the isolated lung epithelium against compression parallel to the distal-proximal axis. The color scale corresponds to that in Figure 3B. The time origin indicates the timing of compression. Scale bar, $30 \mu \mathrm{m}$.

1026

1027

Movie 5 ERK activity response of the isolated lung epithelium to compression vertical to the distal-proximal axis

1029 Time-lapse video of ERK activity at the tip of an isolated lung epithelium against the 1030 compression vertical to the distal-proximal axis. The color scale corresponds to that in Figure 3C. The time origin indicates the timing of compression. Scale bar, $30 \mu \mathrm{m}$.

1034 Time-lapse video of ERK activity at the tip of the isolated lung epithelium after treatment with $10351 \mu \mathrm{M}$ PD0325901. The color scale corresponds to that in Figure S4A. Time origin indicates 1036 the timing of inhibitor administration. Scale bar, $30 \mu \mathrm{m}$. 
1038 Movie 7 Response of ERK activity and F-actin accumulation to acute simulation with

1039 FGF1

1040 Time-lapse video of ERK activity (left) and SiR-Actin signal (right) at the tip of the isolated

1041 lung epithelium after treatment with $500 \mathrm{ng} \mathrm{mL}^{-1} \mathrm{FGF}$. The color scales correspond to those

1042 in Figure 5A. The time origin indicates the timing of FGF1 administration. Scale bars, $30 \mu \mathrm{m}$.

1044 Movie 8 Simulation for the morphogenesis of the proliferating epithelial monolayer in the

1045 regime of curvature-dependent apical extension

1046 Colors represent the sheet curvature. The color scale corresponds to that in Figure 6E. Scale

1047 interval of the graph is $20 \mu \mathrm{m}$.

1048

1049 Movie 9 Simulation of virtual epithelial monolayer with a short retention time for 1050 curvature memory, $\gamma=1$

1051 Colors represent the cellular F-actin levels. The color scale corresponds to that in Figure $6 \mathrm{H}$.

1052 Scale interval of the graph is $20 \mu \mathrm{m}$.

1053

1054 Movie 10 Simulation of virtual epithelial monolayer with a medium retention time for

1055 curvature memory, $\gamma=\mathbf{1 0}^{-3}$

1056 Colors represent cellular F-actin levels. The color scale corresponds to that in Figure $6 \mathrm{H}$. Scale

1057 interval of the graph is $20 \mu \mathrm{m}$.

1058

1059 Movie 11 Simulation of virtual epithelial monolayer with a long retention time for 1060 curvature memory, $\gamma=\mathbf{1 0}^{-6}$

1061 Colors represent cellular F-actin levels in. The color scale corresponds to that in Figure $6 \mathrm{H}$.

1062 Scale interval of the graph is $20 \mu \mathrm{m}$. 
bioRxiv preprint doi: https://doi org/10.1101/2021.07 11.451982; this version posted July 12, 2021. The copyright holder for this preprint (which was not certified by peer review) is the author/funder, who has granted bioRxiv a license to display the preprint in perpetuity. It is Figure 1 made available under aCC-BY-NC-ND 4.0 International license.

A

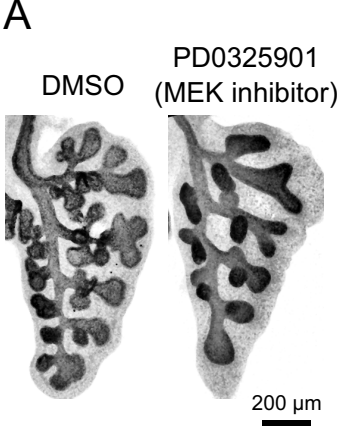

$E$

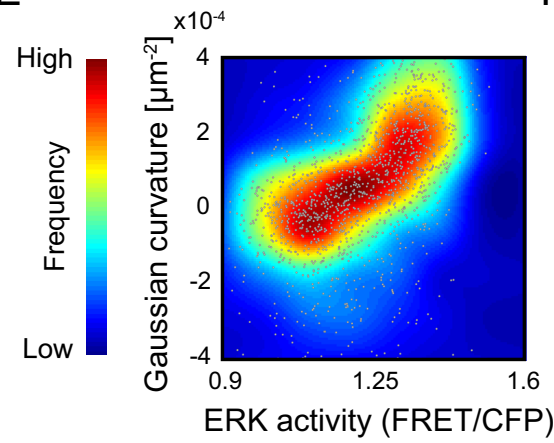

$B$

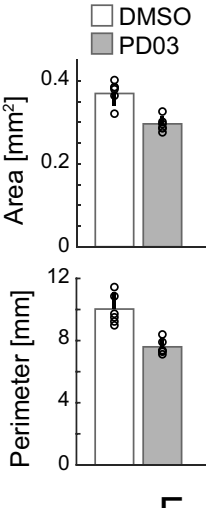

$\mathrm{F}$

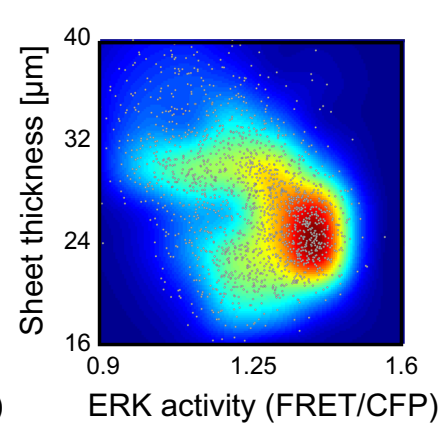

C

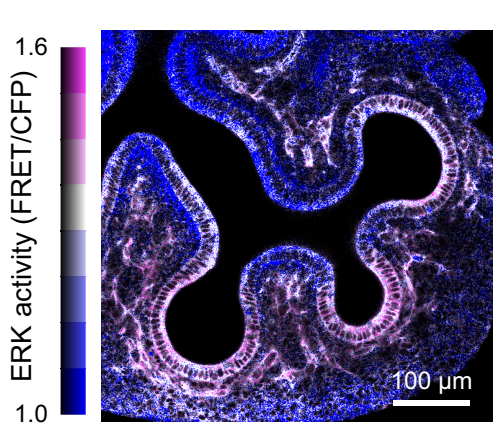

G

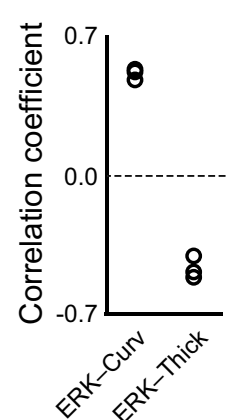

D

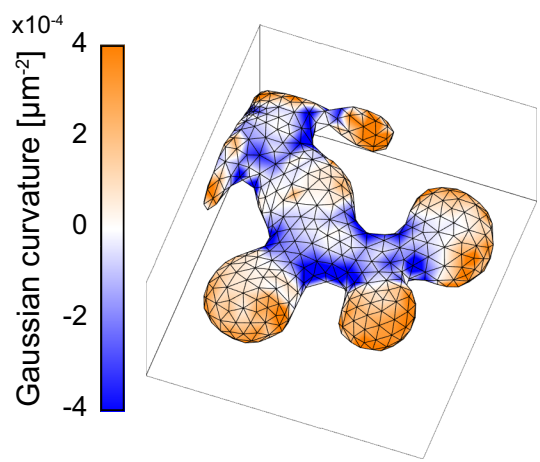

$\mathrm{H}$

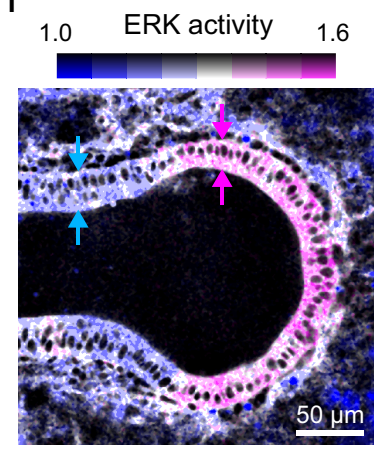


bioRxiv preprint doi: https://doi org/101101/2021 07 11.451982; this version posted July 12, 2021. The copyright holder for this preprint (which was not certified by peer review) is the author/funder, who has granted bioRxiv a license to display the preprint in perpetuity. It is

Figure 2 made available under aCC-BY-NC-ND 4.0 International license.

A

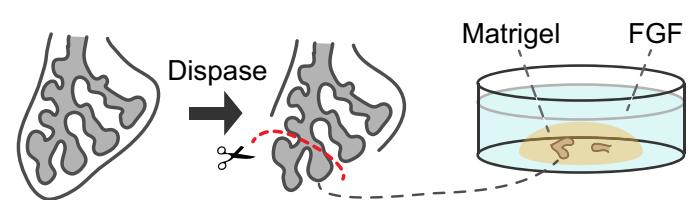

B

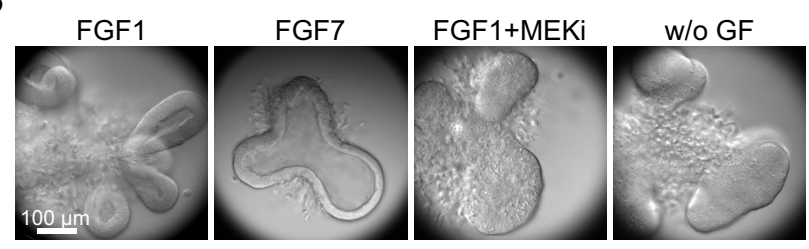

C

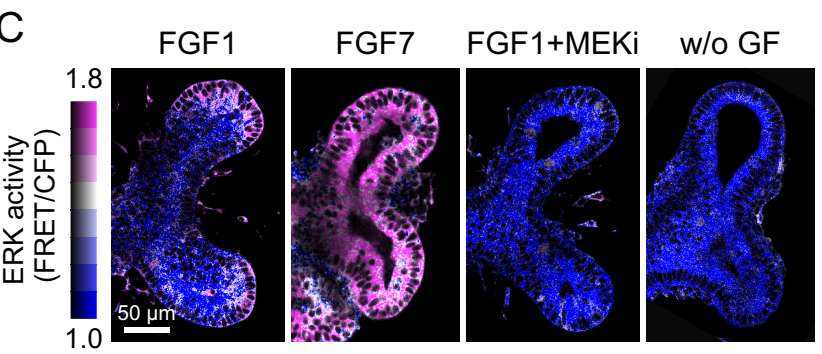

D ERK activity (FRET/CFP)

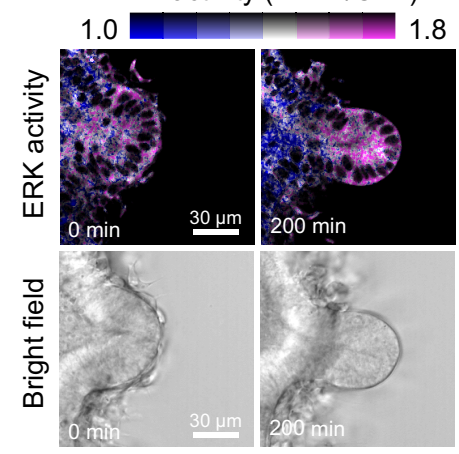

E

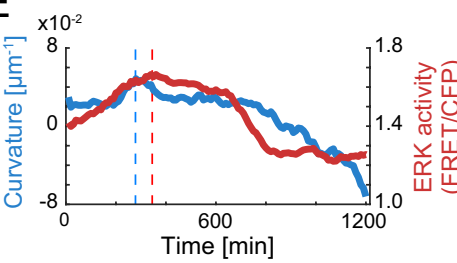

$\mathrm{F}$

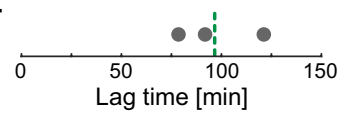

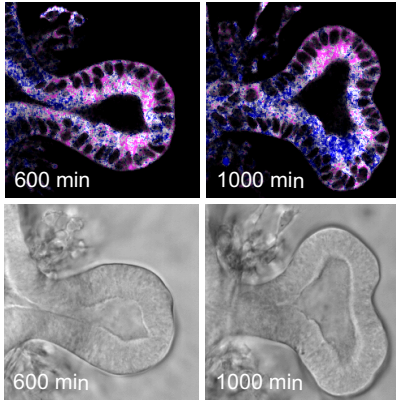

G Basal Apical

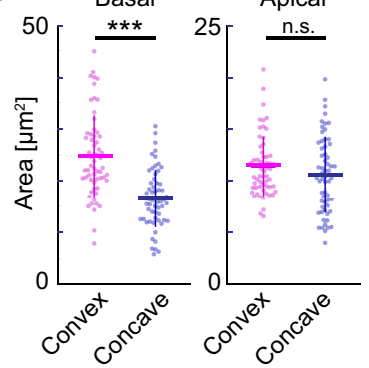


bioRxiv preprint doi: https://doi org/101101/2021 07 11.451982; this version posted July 12 2021. The copyright holder for this preprin (which was not certified by peer review) is the author/funder, who has granted bioRxiv a license to display the preprint in perpetuity. It is

Figure 3 made available under aCC-BY-NC-ND 4.0 International license.

A

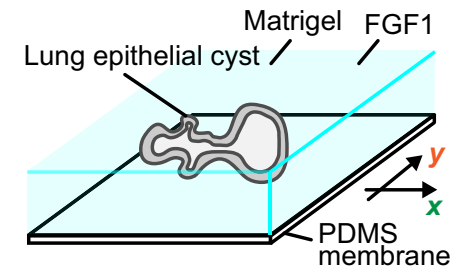

$\mathrm{D}$

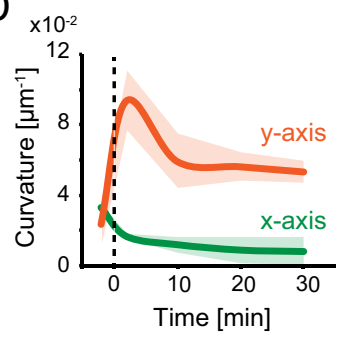

$\mathrm{F}$

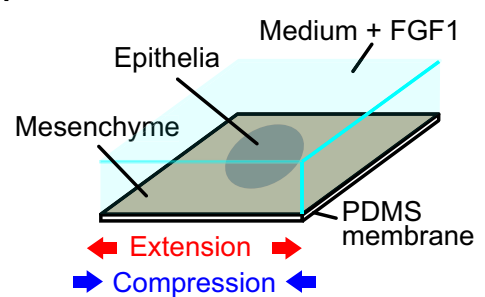

$\mathrm{E}$

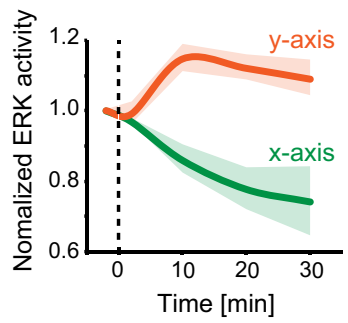

G

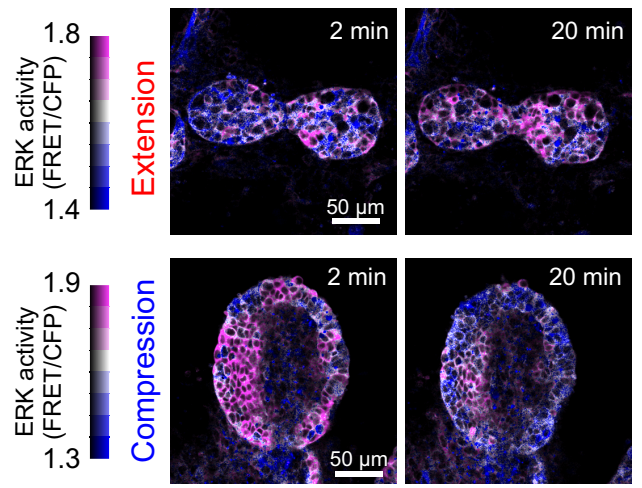

C
B
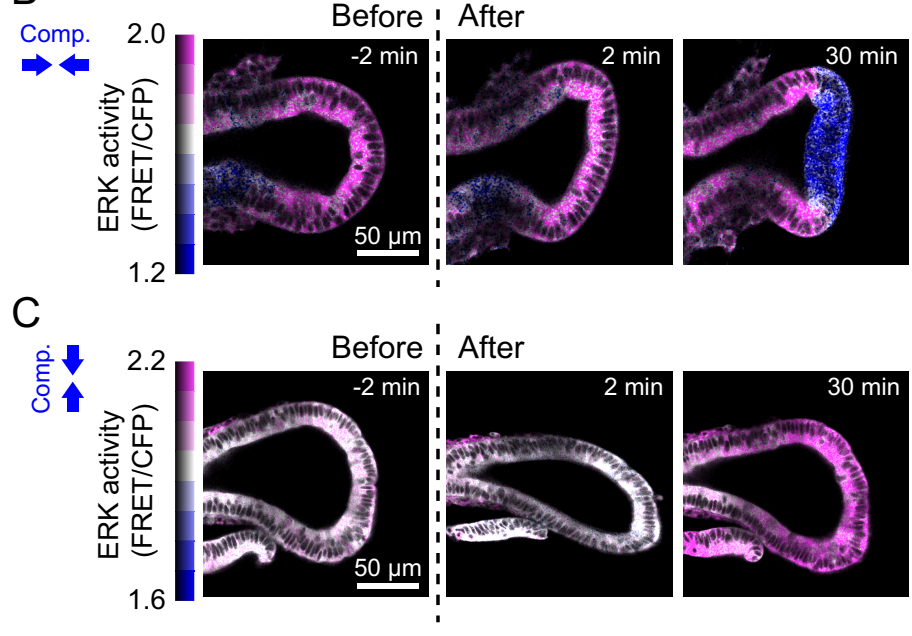

$\mathrm{H}$

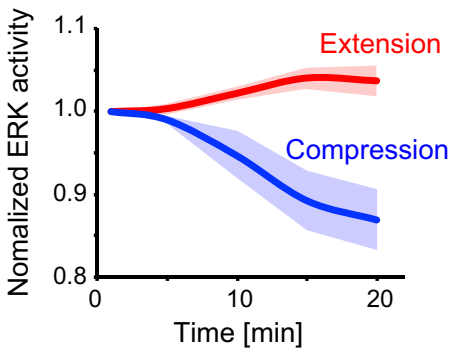


bioRxiv preprint doi: https://doi org/101101/2021.07.11.451982; this version posted July 12, 2021. The copyright holder for this preprin (which was not certified by peer review) is the author/funder, who has granted bioRxiv a license to display the preprint in perpetuity. It is

Figure 4 made available under aCC-BY-NC-ND 4.0 International license.

A

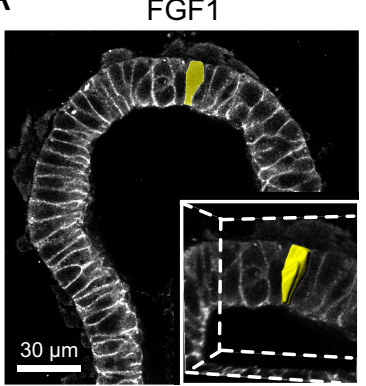

D

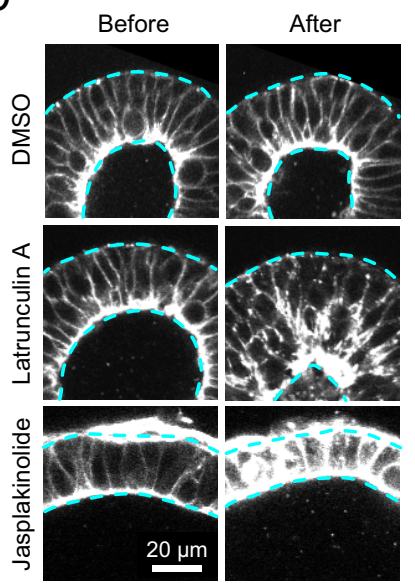

FGF1 + MEK inhibitor

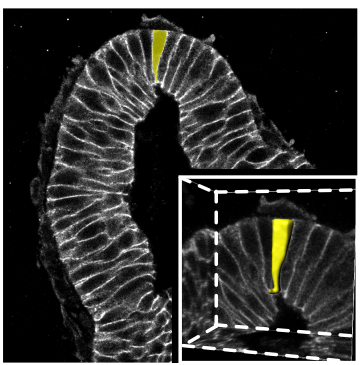

E

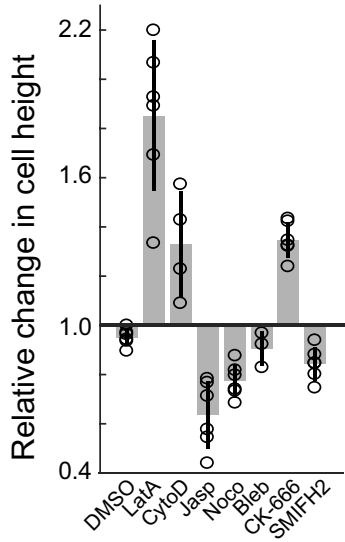

B

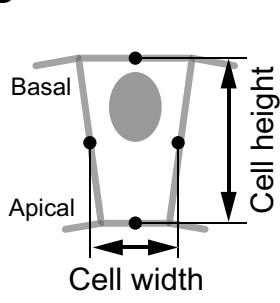

F
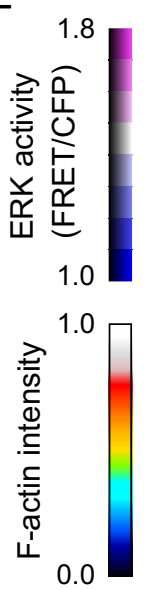
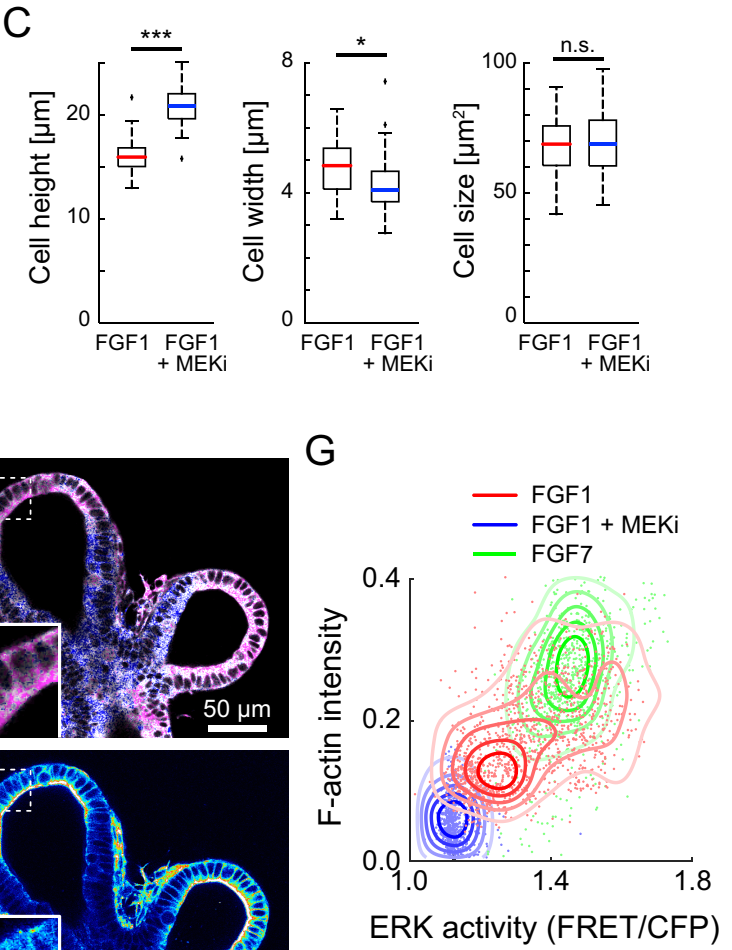
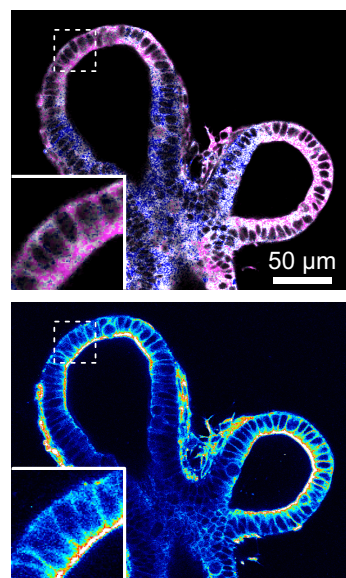
bioRxiv preprint doi: https://doi org/101101/2021 07 11.451982; this version posted July 12 2021. The copyright holder for this preprin (which was not certified by peer review) is the author/funder, who has granted bioRxiv a license to display the preprint in perpetuity. It is

Figure 5 made available under aCC-BY-NC-ND 4.0 International license.

A
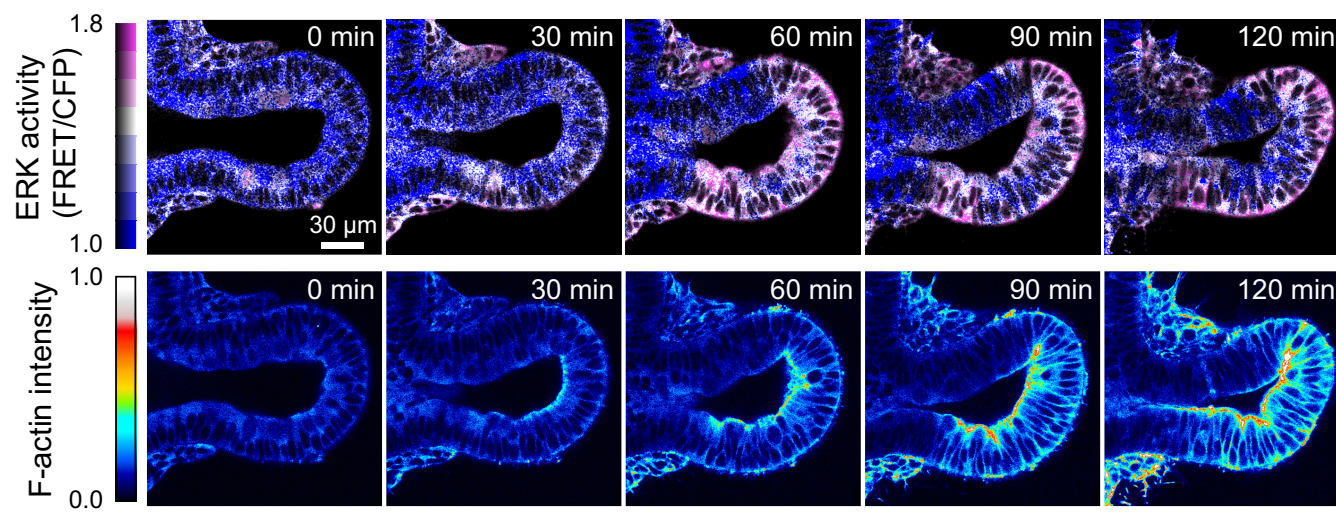

B

C
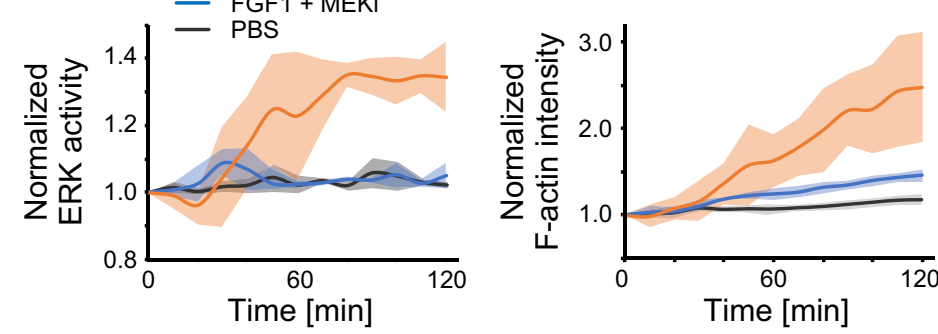

D

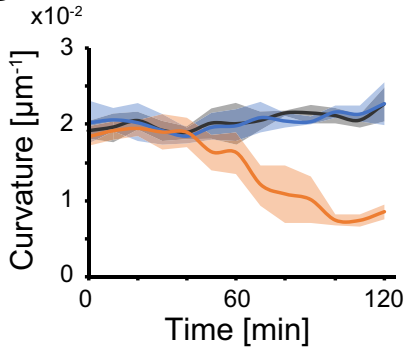


bioRxiv preprint doi: https://doi org/101101/2021.07 11.451982; this version posted July 12, 2021. The copyright holder for this preprint (which was not certified by peer review) is the author/funder, who has granted bioRxiv a license to display the preprint in perpetuity. It is Figure 6 made available under aCC-BY-NC-ND 4.0 International license.

A

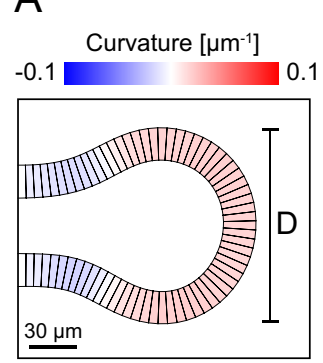

D

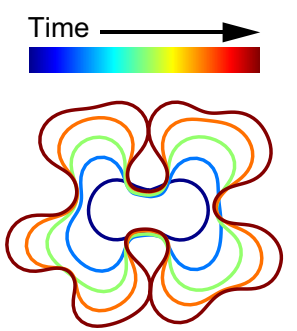

$\mathrm{H}$
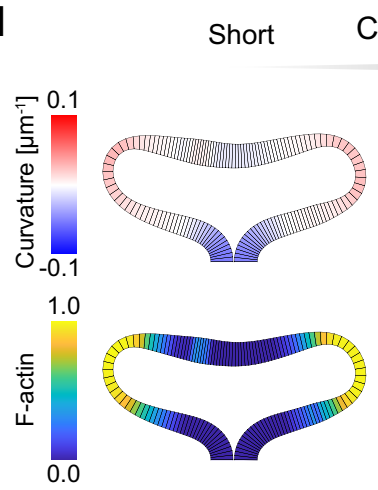

$B$

E
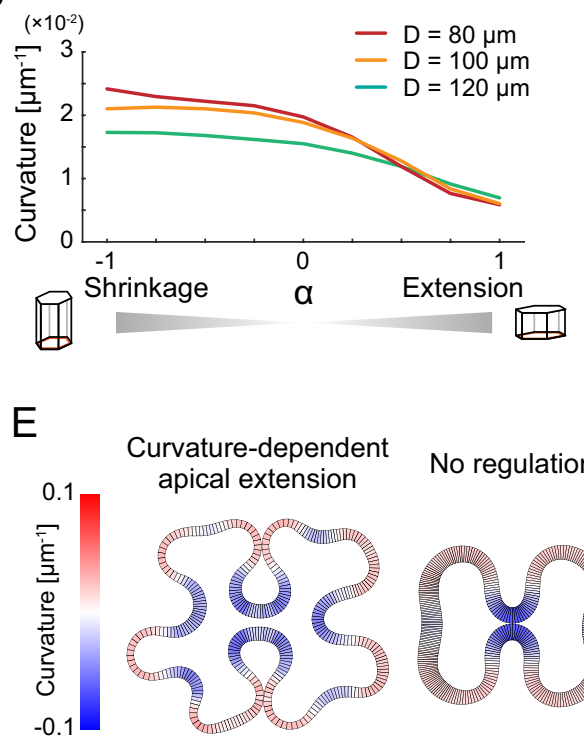

Curvature memory time $\left(\mathrm{\gamma}^{-1}\right)$ Long
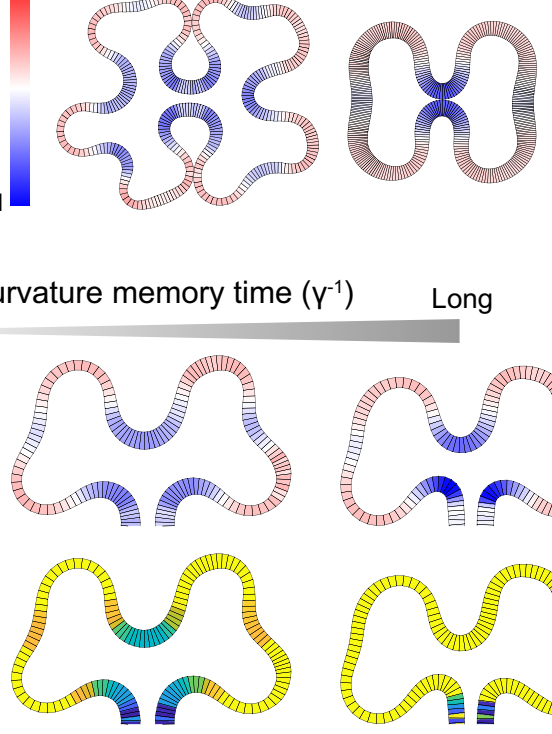

C

Curvature-dependent No regulation Curvature-dependent
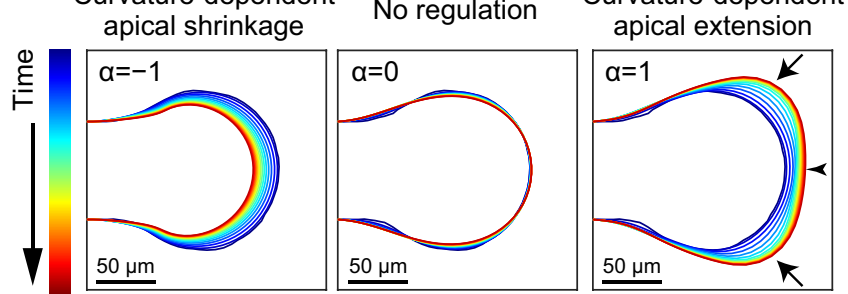

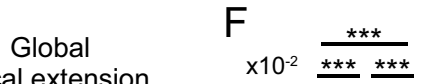

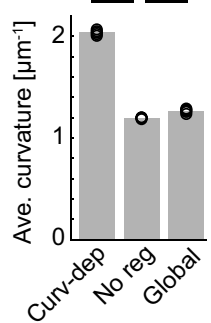

G
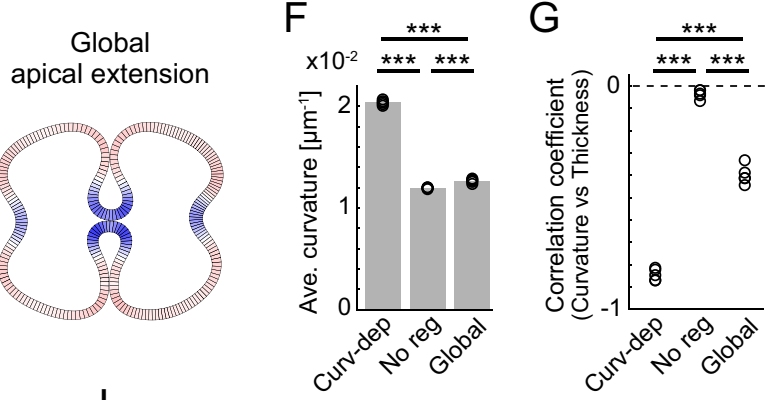

8

I

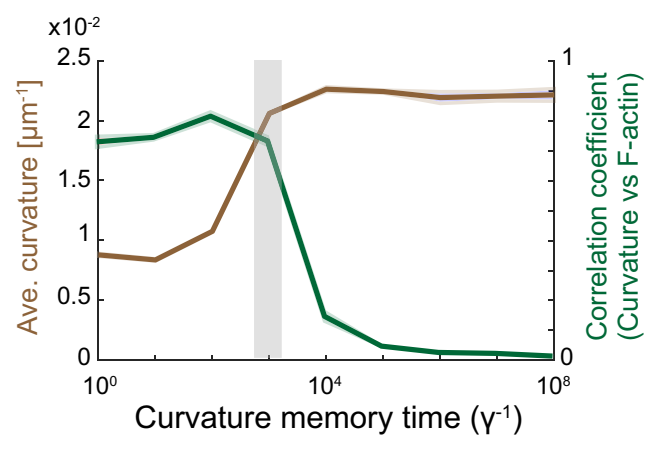


bioRxiv preprint doi: https://doi.org/101101/2021.07.11.451982; this version posted July 12, 2021. The copyright holder for this preprint (which was not certified by peer review) is the author/funder, who has granted bioRxiv a license to display the preprint in perpetuity. It is

Figure 7 made available under aCC-BY-NC-ND 4.0 International license.

\section{Curvature-sensitive ERK activation}

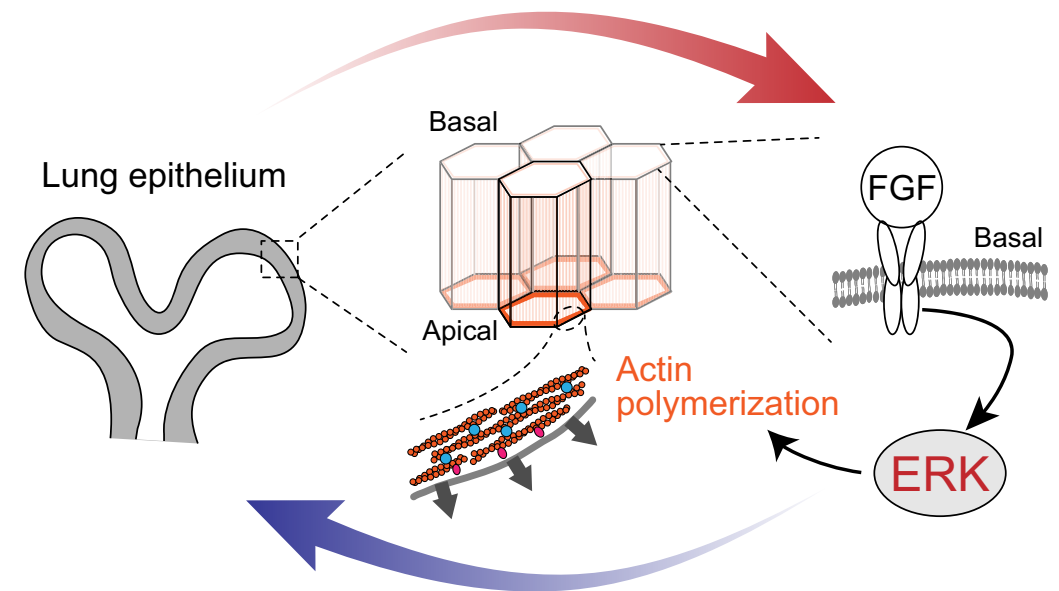

ERK-driven tissue bending 